OPEN ACCESS

Edited by:

Xufeng Jing,

China Jiliang University, China

Reviewed by:

Jifeng Liu,

Dartmouth College, United States

Venu Gopal Achanta,

Tata Institute of Fundamental

Research, India

${ }^{*}$ Correspondence:

Li-yang Shao

shaoly@sustech.edu.cn

Specialty section:

This article was submitted to

Optics and Photonics,

a section of the journal

Frontiers in Physics

Received: 22 July 2020

Accepted: 30 September 2020

Published: 29 January 2021

Citation:

Hu J, Bandyopadhyay S, Liu Y, Shao L (2021) A Review on Metasurface: From Principle to Smart Metadevices.

Front. Phys. 8:586087.

doi: $10.3389 /$ fphy.2020.586087

\section{A Review on Metasurface: From Principle to Smart Metadevices}

\author{
Jie Hu, Sankhyabrata Bandyopadhyay, Yu-hui Liu and Li-yang Shao* \\ Department of Electrical and Electronic Engineering, Southern University of Science and Technology, Shenzhen, China
}

Metamaterials are composed of periodic subwavelength metallic/dielectric structures that resonantly couple to the electric and magnetic fields of the incident electromagnetic waves, exhibiting unprecedented properties which are most typical within the context of the electromagnetic domain. However, the practical application of metamaterials is found challenging due to the high losses, strong dispersion associated with the resonant responses, and the difficulty in the fabrication of nanoscale 3D structures. The optical metasurface is termed as 2D metamaterials that inherent all of the properties of metamaterials and also provide a solution to the limitation of the conventional metamaterials. Over the past few years, metasurfaces; have been employed for the design and fabrication of optical elements and systems with abilities that surpass the performance of conventional diffractive optical elements. Metasurfaces can be fabricated using standard lithography and nanoimprinting methods, which is easier campared to the fabrication of the counterpart 3 days metamaterials. In this review article, the progress of the research on metasurfaces is illustrated. Concepts of anomalous reflection and refraction, applications of metasurfaces with the Pancharatanm-Berry Phase, and Huygens metasurface are discussed. The development of soft metasurface opens up a new dimension of application zone in conformal or wearable photonics. The progress of soft metasurface has also been discussed in this review. Meta-devices that are being developed with the principle of the shaping of wavefronts are elucidated in this review. Furthermore, it has been established that properties of novel optical metasurface can be modulated by the change in mechanical, electrical, or optical stimuli which leads to the development of dynamic metasurface. Research thrusts over the area of tunable metasurface has been reviewed in this article. Over the recent year, it has been found that optical fibers and metasurface are coagulated for the development of optical devices with the advantages of both domains. The metasurface with lab-on fiberbased devices is being discussed in this review paper. Finally, research trends, challenges, and future scope of the work are summarized in the conclusion part of the article.

Keywords: metasurface, wavefront modulation, flexible metasurface, reconfigurable metasurface, meta-device, lab on fiber 


\section{INTRODUCTION}

Over the past 2 decades, metamaterials [41, 192] have attracted lots of interests owing to their remarkable electromagnetic features. Metamaterials are arrays of special-shaped scattering elements rationally designed, and exhibit some special electromagnetic responses, for instance, negative-index media [139, 143], zero-index materials [196] and ultra-highindex materials $[117,136]$. The two-dimensional counterpart of metamaterials, metasurface $[74,104,107]$ is much easier to be fabricated and utilized. It can exhibit the amazing capacity of electromagnetic wavefront manipulation, which is mainly introduced by the interaction between an electromagnetic wave and these meta-atoms structures as well as their functional arrangements. Over recent years, metasurface paradigm is considered as an important way to engineer electromagnetic wave, including wavefront shaping [63, 162], polarization conversion [168, 194] and radiation control or energy concentration [18, 79]. With these versatile electromagnetic properties, metasurface has attracted enormous attention from the research communities. Based on strong wavefront modulation capability of metasurface within the sub-wavelength scale, many mete-devices have been demonstrated in the last ten years, such as meta-lens [16, 21, 94, 155], invisible cloak [50, 126], absorber [52, 98, 169], vortex beam generator [176], holography $[43,113,193]$ and so on.

it was observed that the dynamic performance of the metasurface is essential for modern-day science and technological applications but conventional metasurface limits its operation in terms of tunability and reconfiguration. Conventional metasurface provides static and predefined optical functions that are normally being governed by the geometry, shape, and arrangements of meta-atoms. Over the past few years researchers put an effort to develop flexible and reconfigurable metasurfaces, where the shape, size, and arrangements of the meta-atoms can be tuned or altered with external stimuli [33, 45, 85]. And many researchers are devoting themselves to access reprogrammable metasurface [83, 87, 103].

Progressive research on metasurface has enormously expanded its application domain over the past decade or so. In this article, a primary focus is being given to the applications of metasurface through wavefront engineering. We have reviewed recent works in the area of metasurface based arbitrary wavefront modulation, and in due course, the review is being extended with flexible and reconfigurable metasurface and meta-devices. The principles of operation of the device, applications along with their extraordinary advantages, fabrication challenges, and prospects are being discussed in a detailed manner. In the aspect of fabrication of new and novel optical devices, metasurface and optical fiber integrated $[124,125]$ for the development of novel "lab-on-fiber" structures are also illustrated in this paper, which possess enormous potentials for future applications. The review work is concluded with a summarization of the challenges and prospects of this research area.

\section{THE PRINCIPLE OF METASURFACE FOR WAVEFRONT MODULATION}

The regulation of electromagnetic waves with traditional optical components, such as lenses and prisms, is realized through the accumulation of phase delay in the process of light propagation, which greatly limits the reduction and integration of optical devices. Control of phase and amplitude plays a crucial part in wavefront modulation. Those traditional optical elements, as well as diffractive elements such as gratings and holograms, are bulky for optical set-up. Whereas, metasurface can modify the amplitude and impart an abrupt phase shift to the incident wave within the sub-wavelength scale through the light-matter interaction, and thus realize the wavefront modulation much more efficiently. In the past few years, several excellent review papers have summarized the development of this field [34, 165]. There are three main types of mechanism for metasurface to manipulate the phase variation under certain polarization states, including resonances with linearly polarized (LP) light, Pancharatnam-Berry (PB) phase for circularly polarized (CP) light, and Huygens' principle with polarization independence [146].

\section{Wavefront Engineering of Linearly Polarized Light}

Fermat's principle states that light travels along an extremum path. In 2011, Nanfang Yu et al. put forward generalized laws of refraction and reflection in succession, adapted Fermat's principle to a larger class of interfaces where the presence of antennas may provide abrupt phase jump to incident waves [176]. They derived the general relation between an incident and scattered waves as

$$
\left\{\begin{array}{l}
n_{t} \sin \left(\theta_{t}\right)-n_{i} \sin \left(\theta_{i}\right)=\frac{\lambda_{0}}{2 \pi} \frac{d \phi(x)}{d x} \\
n_{i} \sin \left(\theta_{r}\right)-n_{i} \sin \left(\theta_{i}\right)=\frac{\lambda_{0}}{2 \pi} \frac{d \phi(x)}{d x}
\end{array}\right.
$$

where $n_{i}$ and $n_{\mathrm{t}}$ are the refractive indices on the two sides of the interface, $\lambda_{0}$ is the free space wavelength, $\theta_{i}, \theta_{r}$ and $\theta_{t}$ are the incident, reflected, and transmitted angles, as shown in Figure 1A, and $d \phi / d x$ indicates the gradient of the phase discontinuity along with the interface, provided by the metaatoms of the metasurface. Equation 1 implies that both the refracted and reflected beam can have an arbitrary direction, provided that a suitable constant gradient of phase discontinuity along the interface $(d \phi / d x)$ is introduced. By engineering a phase discontinuity along an interface, one can fully steer light and accomplish unparalleled control of anomalous reflection and refraction described by the generalized Snell's law. Implementation of this idea was accomplished by $\mathrm{V}$-shaped gold nanoantennas which were used as a gradient metasurface. As shown in Figure 1B, V-shaped resonators support "symmetric" and "antisymmetric" modes, which are excited by electric-field components along $\hat{s}$ and $\hat{a}$ axes, respectively. By changing the length and the opening angle of plasmonic nanorods, one can get an abrupt phase shift over a $\pi$ phase range as well as different amplitudes, as shown in Figure 1C. 
A

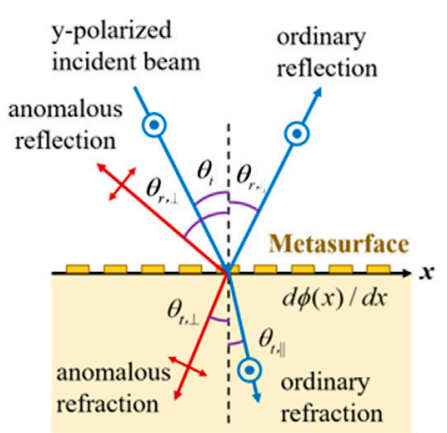

B

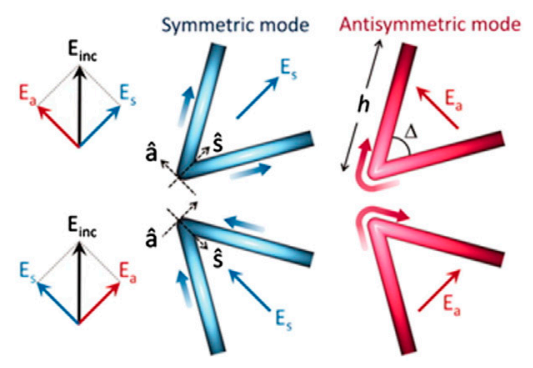

C
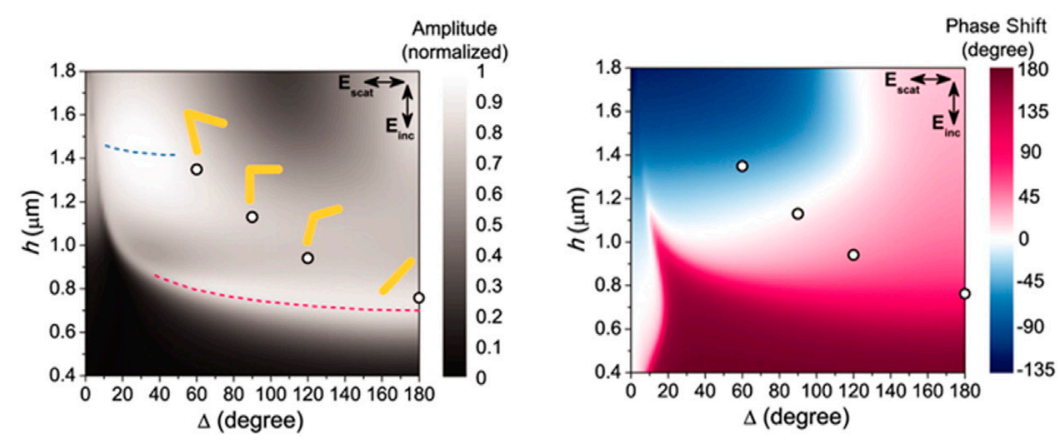

FIGURE 1 | (A) Generalized Snell's law of refraction and reflection: Schematic of anomalously refracted and reflected beams for cross-polarized scattered beams. (B) $V$-shaped nanoantenna supports symmetric and antisymmetric modes, which are excited by cross-polarized components of the incident light. There is a $\pi$ phase difference between the mirror structures [176]. (C) Simulated amplitude and phase shift of the cross-polarized scattered light for V-shaped nanoantennas with different length and opening angle of gold nanorods at wavelength of $8 \mu \mathrm{m}$ [176]. (D) Anomalous refraction phenomenon caused by phase discontinuities with a gradient metasurface consisting of $\mathrm{V}$-shaped nanoantenna array [176]. (E) Anomalous refraction phenomenon caused by metasurface operating in reflection with a back ground plane [141]. Figure reproduced with permission from: (B)-(D), ${ }^{\circledR}$ 2011, American Association for the Advancement of Science (AAAS); (E), ${ }^{\circ}$ 2012, American Chemical Society (ACS).

However, to achieve full phase regulation, the phase shift range must be no less than $2 \pi$ phase coverage. Yu et al. realized that, for linearly polarized incident light, a $90^{\circ}$ rotation of the $\mathrm{V}$-shaped antennas allowed the remaining $\pi$ range to be accessed in the cross-polarized scattered waves [111, 175, 176]. As shown in Figure 1D, if each second source at a position $x$ of the interface carries an abrupt phase variation over $2 \pi$ phase coverage, the abnormal refraction and reflection can be observed. Reference to the theory of gradient metasurface, many other structure designs such as C-shaped antennas [96], nanorods [58] and nanobricks [121], were proposed later and demonstrated to have full phase control capability. What's more, by adjusting the size of the unit cell, these electromagnetic responses were achieved in different frequency ranges, such as visible-band [93], near-infrared [74] and mid-infrared frequencies [176]. However, these proposed designs were realized by cross-polarized components coupled to the scattering field. The maximum coupling efficiency between the two polarizations was extremely limited within a single metasurface, and thus, only a small amount of incident electromagnetic energy can interact with the metasurface while a large amount of energy radiated outside in an ordinary way [109]. In order to improve the efficiency, gradient metasurfaces operating in reflection with a back ground plane were proposed $[121,122,141]$, as shown in Figure 1E. This type of metasurface consisted of metallic nanoantennas array separated from a metallic ground film with a thin dielectric layer, namely Metal-Insulator-Metal (MIM) structures. Thanks to the strong coupling between the top antennas layer and the ground metallic plane, magnetic resonances as gap modes inside the dielectric spacer were excited, and thus phase delay up to $2 \pi$ can be achieved.

\section{Modualtion of Circularly Polarized Light Through Pancharatnam-Berry Phase}

The metasurfaces discussed in the previous subsection are designed for linearly polarized waves, which is incapable for controlling CP waves. Recently, Pancharatnam-Berry phase metasurfaces [57] attracted intense attention due to their strong capabilities in modulating $\mathrm{CP}$ waves. Consider two arrays of identical scattering elements placed on the $x-y$ plane, and the structures in one group are rotated at an angle of $\theta$ with respect to the counterparts in the other group. Then illuminating the two scatter arrays by the same CP beam, only one difference can be observed between the spin-flipped components of waves scattered by these two arrays of meta-atoms, which is a phase factor $e^{i 2 \theta}$, independent of the scatter details and the frequency. This phenomenon is called $\mathrm{PB}$ phase, which can be created by any anisotropic optical antennas. From the mathematical derivation, assuming that the incident $\mathrm{CP}$ wave is left-handed circular 
polarized (LCP) light $\mathrm{E}_{i}=E_{L C P}=\left({ }^{1}\right)$ (or $=E_{R C P}=\left({ }^{1}\right)$ for righthanded circular polarized (RCP) light), the scattered light $E_{\mathrm{s}}$ from an anisotropic antenna with an orientation angle of $\theta$ can be described as Refs. 49 and 174.

$$
\begin{aligned}
E_{s} & =\left(\begin{array}{cc}
\cos \theta & -\sin \theta \\
\sin \theta & \cos \theta
\end{array}\right)\left(\begin{array}{cc}
t_{o} & 0 \\
0 & t_{e}
\end{array}\right)\left(\begin{array}{cc}
\cos \theta & \sin \theta \\
-\sin \theta & \cos \theta
\end{array}\right) \times E_{i} \\
& =\frac{t_{o}+t_{e}}{2} \mathrm{E}_{L C P}+\frac{t_{o}-t_{e}}{2} \exp ( \pm \mathrm{i} 2 \theta) \mathrm{E}_{R C P}
\end{aligned}
$$

where $t_{o}$ and $t_{e}$ are the scattering coefficients for the two orthogonal linear polarization components of the incident light along the two axes of the scattering elements. From the Eq. 2, it is clear that the scattered light consists of two circular polarization states: one component has the same handedness as the incident CP beam without any phase delay (as shown by the first term of the above equation), and the other component is cross-polarization with a phase delay of $\pm 2 \theta$ (as shown by the second term of the above equation). The sign "+" and "-" represent the phase delay for the incident LCP and RCP light, respectively, as shown in Figure 2A [57]. Because the phase delay is linear to the orientation angle of the scattering elements, it can provide continuous phase control from 0 to $2 \pi$ very easily, by rotating the anisotropic meta-atoms from 0 to $\pi$, such as C-shaped split-ring resonators, nanorods, and nanoslits [58, 145, 179]. Although $\mathrm{PB}$ phase metasurface can regulate $\mathrm{CP}$ beams, the conversion efficiencies of earlier published works were very low. It was demonstrated that the maximum conversion efficiency of a single layer ultrathin $\mathrm{PB}$ phase metasurface was limited by $25 \%$ [31]. To improve the working efficiency, MIM structures working in reflection style were proposed and proved to be an effective approach [193]. On the other hand, if the metasurface contains both electric and magnetic resonators, resulting in constructive interference at the transmission side and destructive interference at the reflection side, and thus a transmissive ultrathin $\mathrm{PB}$ metasurface with an ultra-high efficiency can be achieved [102] as shown in Figure 2B.

\section{Huygens' Principle With Polarization Independence}

Different from those designs based on phase compensation on different polarization states mentioned above, Huygens' metasurface considers the impedance matching on the interface with polarization insensitivity [116], and simultaneous excitation of electric and magnetic dipole moments are achieved. According to the surface equivalence principle $[116,133]$, it was understood that both electric and magnetic surface currents were required on the interface to create the desired field distribution and meet the boundary conditions, as shown in Figure 2C. Consequently [116],

$$
\left\{\begin{array}{l}
\vec{J}_{s}=\widehat{n} \times\left(\vec{H}_{2}-\vec{H}_{1}\right) \\
\vec{M}_{s}=-\widehat{n} \times\left(\vec{E}_{2}-\vec{E}_{1}\right)
\end{array}\right.
$$

where $\vec{J}_{s}$ and $\vec{M}_{s}$ are the required electric and magnetic surface currents. The specially designed subwavelength metallic texture was usually fabricated on the dielectric substrate to get the required surface impedance of each unit. A general method was proposed to convert the desired transmission/reflection phase distributions to the required surface profiles of Huygens' metasurface [108]. Minimization of the transmission loss was accomplished by high contrast transmits/reflect arrays and it was developed by using a significant high index layer $[4,5]$.

In particular, metallic metasurface has a large ohmic loss in optical frequencies, which significantly limits and even degrades its performance. Thus, in this regime, high-index dielectric resonators with low loss (dielectric metasurface) was a hot topic in this decade $[6,93,167,177]$. Electric and magnetic resonances are excited in dielectric metasurface, which can be classified as Mie resonances $[36,39,53,115]$, replacing the plasmonic resonances in metallic counterparts, and thus cause the dielectric metasurface to produce electromagnetic response, including amplitude modulation, abrupt phase jump production and so on $[4,134]$.

\section{THE APPLICATIONS: WAVEFRONT ENGINEERING WITH METASURFACE}

Since the advent of the metasurface, it has demonstrated excellent wavefront shaping ability, which makes it a promising application in many fields. After sorting the theory and techniques of metasurface to control the phase and amplitude under different polarization states, a detailed review of their applications in the domain of photonics devices is being discussed in this section.

\section{Meta-Lens}

Metasurface can provide abrupt phase mutations to incident electromagnetic waves within sub-wavelength scales. In the previous section, we have discussed the abnormal refraction and reflection of incident light caused by these abrupt phase variations along the interface of the two medium. The light beam can be twisted, focused; and special optical beams like optical vortex beam [10, 134, 178], and Bessel beam [14] can be generated with a controlled phase profile of the metasurface. Phase profile engineering is the basic tool that has being used over the years to control the shape of the incident wave and a detailed discussion over creation of the phase profile for meta-lens are being given in this section.

Assuming the electromagnetic energy was focused at a distance of " $f$ ", as shown in Figure 3A, the phase distribution (in the $\mathrm{x}-\mathrm{y}$ plane) for transmitted or reflected wave should be arranged as the Eq. 4 below [174] to compensate for the optical path difference:

$$
\phi(x, y)=\frac{2 \pi}{\lambda}\left(\sqrt{x^{2}+y^{2}+f^{2}}-f\right)
$$

Based on the concept of optical phase discontinuities, Capasso et al. demonstrated a meta-lens at Telecom wavelength with $\mathrm{V}$-shaped nanoantennas, as shown in Figure 3A [2]. However, the focusing efficiency was approximately $1 \%$ only. Complementary $\mathrm{V}$-shaped apertures were also proposed to 

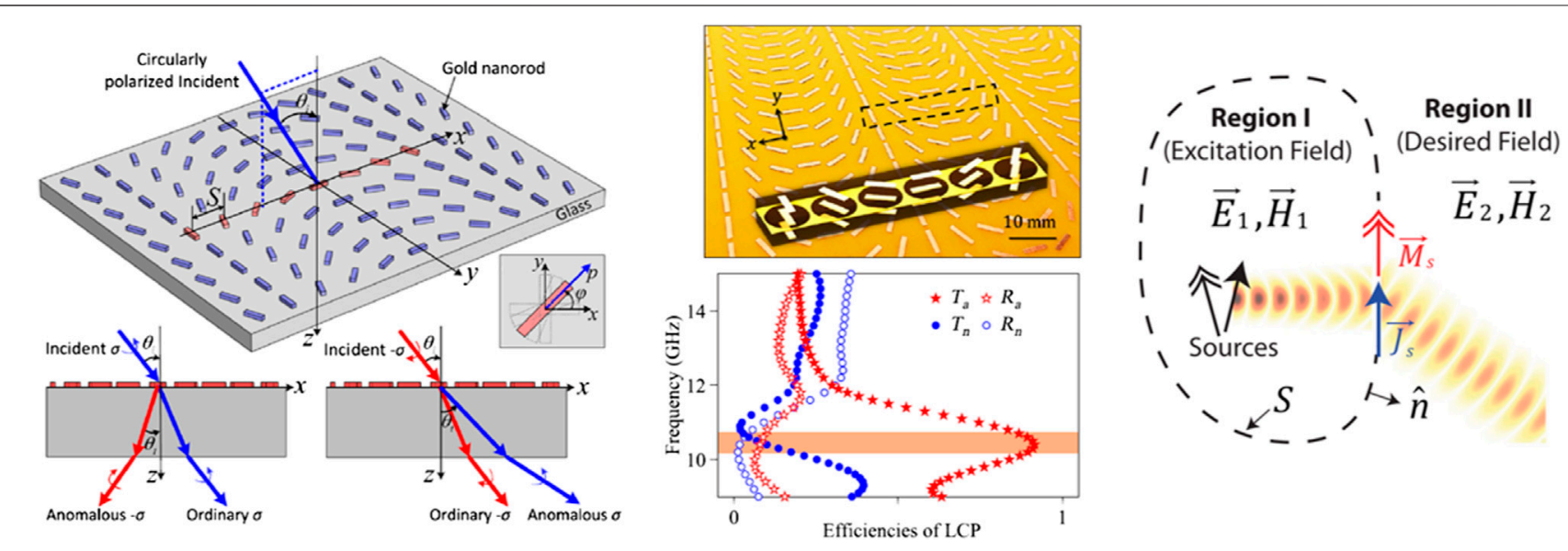

FIGURE 2 | (A) Schematic diagram of normal and anomalous refraction by Pancharatnam-Berry metasurface when illuminated by LCP and RCP light, respectively, [57]. (B) Picture of fabricated Pancharatnam-Berry metasurface exhibiting both electric and magnetic responses and its ultra-high transmissive conversion efficiency [102]. (C) The surface equivalence principle is employed to find the fictitious electric and magnetic surface currents that satisfy the boundary conditions [116]. Figure reproduced with permission from: (A), ${ }^{\odot}$ 2012, American Chemical Society (ACS); (B), ${ }^{\odot} 2017$, American Physical Society (APS); (C), ${ }^{\circ}$ 2013, American Physical Society (APS).

realize meta-lens in the optical regime, but the efficiency was not improved [112]. MIM structure-based meta-lens were demonstrated to improve the focusing efficiency, in the near infrared [123] and mid-infrared regimes [183]. Because of the large ohmic loss of metal in optical frequencies, which limits the efficiency to a certain extent, many metallic meta-lens were designed to work in larger wavelength regime, such as Terahertz, millimeter-wave or microwave regime [24, 27, 92].

Recently, dielectric metasurfaces were developed to circumvent the significant Ohmic losses at optical frequencies [89, 173, 189, 197]. Our group compared the resonance performance in metallic and all-dielectric metasurface in a detailed way [51]. In 2014, utilizing the spin-dependent properties of $\mathrm{PB}$ phase, Lin et al. proposed a transmissive metalens composed of thousands of $\mathrm{Si}$ nanobeam nanoantennas. Via rational structural tuning, the metalens was designed to exhibit a focal length $100 \mu \mathrm{m}$ with an NA $=0.43$ at wavelength of $500 \mathrm{~nm}$. When shining the metalens with RCP light through the substrate, the transmissive light was converted to LCP focal spot with a size of $670 \mathrm{~nm}$, which was close to diffraction limit [93]. Later on, Capasso et al. successfully realized the fabrication of high-aspect-ratio titanium dioxide $\left(\mathrm{TiO}_{2}\right)$ metasurface, as shown in Figure 3B. Based on the geometric $\mathrm{PB}$ phase, they demonstrated three metalenses with large NA (0.8) working at wavelengths of 660,532 , and $405 \mathrm{~nm}$ with corresponding efficiencies of 66,73 , and $86 \%$ [72]. In their following work, a polarization-insensitive metalens working at these three wavelengths was demonstrated, which had large NA (0.85) and high efficiency (over 60\%). Importantly, the focusing ability of the incident light was down to diffraction-limited spots $(\sim 0.64 \lambda)$, which were very promising [73].

Since these metalenses operate based on the phase jump governed by resonance conditions, the dispersion effect of the metalens limits their operation in a broad range of frequency. Thus, minimization or removal of chromatic aberrations of metalenses has been a serious matter of concern in the case of designing and fabrication of the metalens. Avayu et al. designed a multi-layer structure by vertically stacking independent metasurfaces and demonstrated a triply working wavelength achromatic metalens in the visible range (450, 550, and $650 \mathrm{~nm}$ ) [9]. Capasso and his co-workers combined multiple dielectric resonators into meta-atoms and realized an achromatic metalens at three discrete wavelengths $(1,300,1,550$ and, $1,800 \mathrm{~nm}$ ) [71]. Similarly, Yu et al. utilized this combining method with new meta-unit geometries that can fulfill the space to a much greater degree, and realized a polarizationindependent metalens with focusing efficiencies over $20 \%$ within a broadband of 1,200-1,650 nm [137], as shown in Figure 3C. Employing both low-Q-factor resonance induced phase and geometric phase that compensate with each other, an achromatic metalens working in a large wavelength band $(1,200-1,680 \mathrm{~nm})$ with the efficiency of $\sim 12 \%$ was demonstrated experimentally [156]. Achromatic metalens consists of GaNbased integrated resonator meta-atom was designed and fabricated, which proved to eliminate chromatic aberration in visible wavelength $(400-660 \mathrm{~nm})$ with an average efficiency of $\sim 40 \%$ [155]. Recently, tunable dielectric meta-lenses were also proposed $[6,68]$ to provide more usage of meta-device with higher freedom. In the later part of this review, we will discuss about these reconfigurable metasurface.

\section{Metasurface Holography}

Metasurface can also realize the holograms by tailoring the amplitude and phase of the electromagnetic wave simultaneously. In 2013, Shalaev et al. proposed a complementary V-shaped groove array fabricated in a $30 \mathrm{~nm}$ gold film on a glass substrate and demonstrated the holographic image of the word "PURDUE" illuminated by a visible laser of $676 \mathrm{~nm}$, as shown in Figure 4A [113]. Based on PB phase arrangement, three-dimensional holography for a jet was 

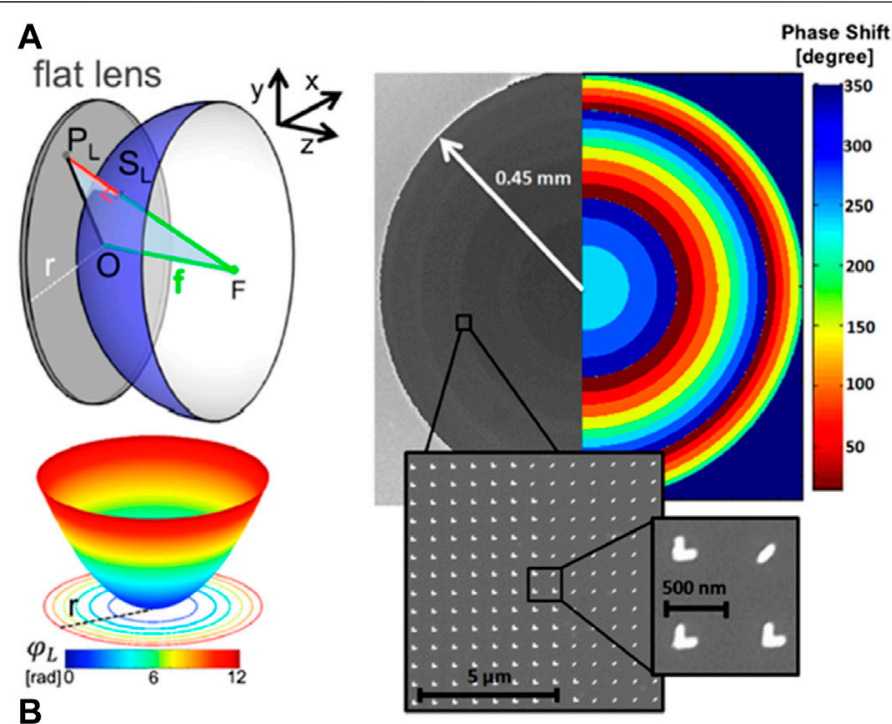

B
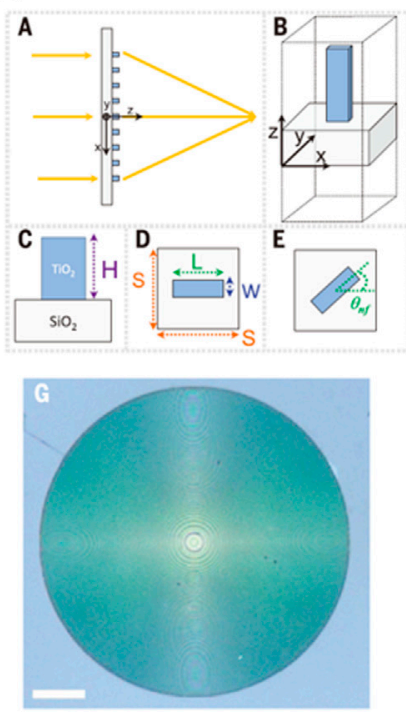
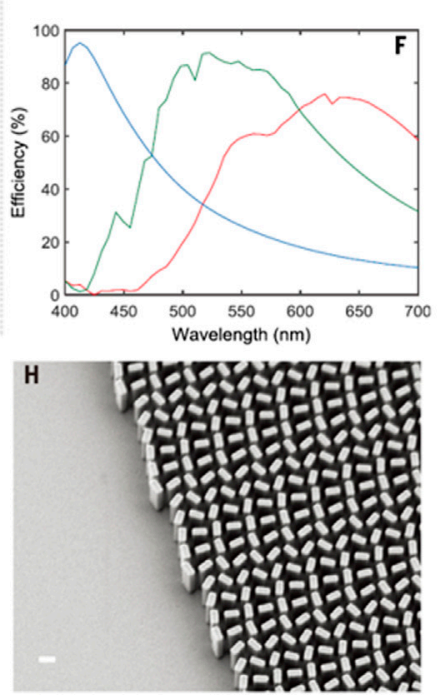

C
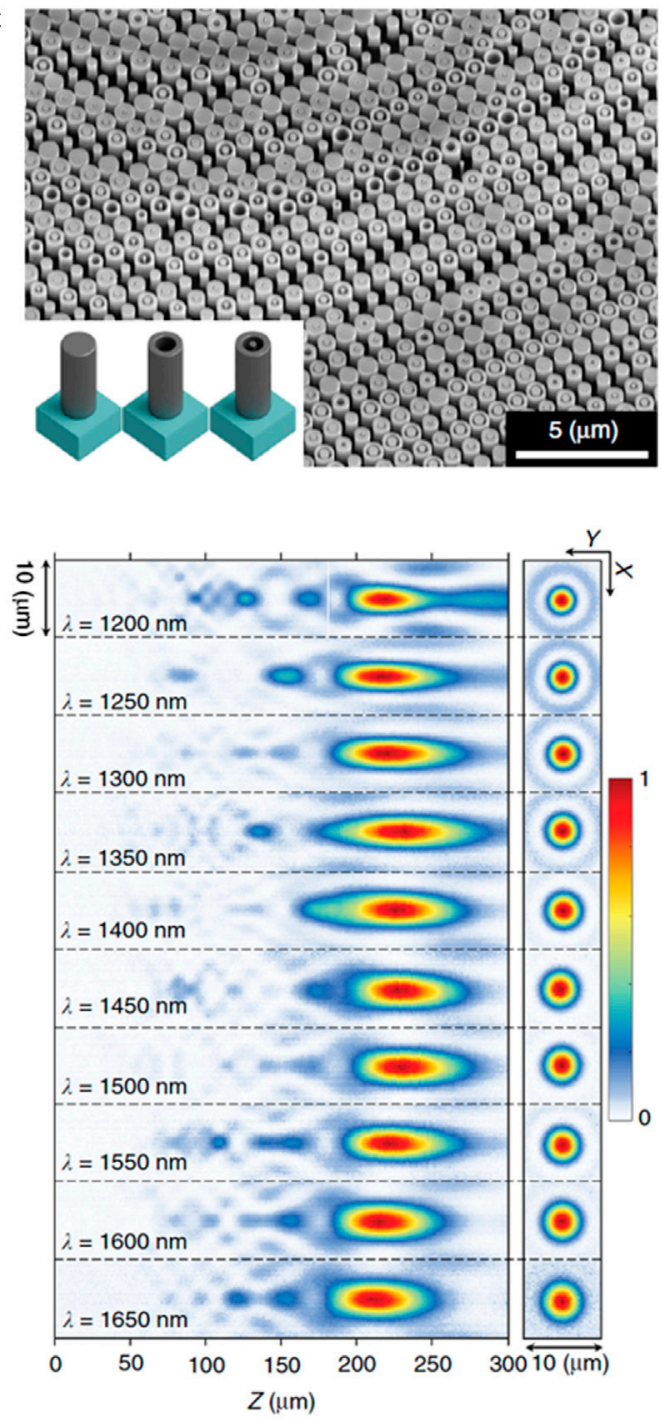

FIGURE 3 | (A) Design schematic diagram of phase distribution of metalens as well as metallic metalens consists of $V$-shaped nanoantennas with different lengths and opening angles of plasmonic nanorods [2]. (B) Dielectric metalens consists of high-aspect-ratio titanium dioxide (TiO2) nanobricks with different orientations [72]. (C) Dielectric metalens working in a large wavelength range $(1,200-1,650 \mathrm{~nm})$ with super meta-atoms composed of multi-resonators [137]. Figure reproduced with permission from: (A), ${ }^{\odot}$ 2012, American Chemical Society (ACS); (B), ${ }^{\odot}$ 2016, American Association for the Advancement of Science (AAAS); (C), ${ }^{\odot}$ 2018, Nature Publishing Group (NPG).

experimentally demonstrated with metallic nanorods metasurface, which had a large field of view estimated as $40^{\circ}$ [58]. Single-layered plasmonic metasurfaces generally suffered from low efficiency, MIM reflection configuration was utilized and realized a high efficiency (80\%) reflective metasurface hologram of Einstein's portrait at the working wavelength of $825 \mathrm{~nm}$, as shown in Figure 4B [193]. Another method to improve the efficiency of the transmissive metasurface hologram is using the dielectric Huygen's metasurface, which has been experimentally demonstrated to reach ultra-high diffraction efficiency over $99 \%$ at the wavelength of $1,600 \mathrm{~nm}$ with silicon nanopillar design [152].
These metasurface holograms mentioned above, in which the meta-atoms were designed for one specific wavelength, were single-color holograms. By combining the multiple resonators with various resonance wavelengths into each meta-unit cell, the matasurface designs can control multi-wavelength beam simultaneously. Based on this methodology, Tsai and coworkers realized binary-phase holograms working for three primary colors (red, green, and blue) with aluminum MIM design, as shown in Figure 4C [60]. Similarly, Choudhury et al. designed super-unit cells consisting of three silver nanoslits with different sizes and orientations, and thus rebuilt transmissive three-color hologram [26]. 

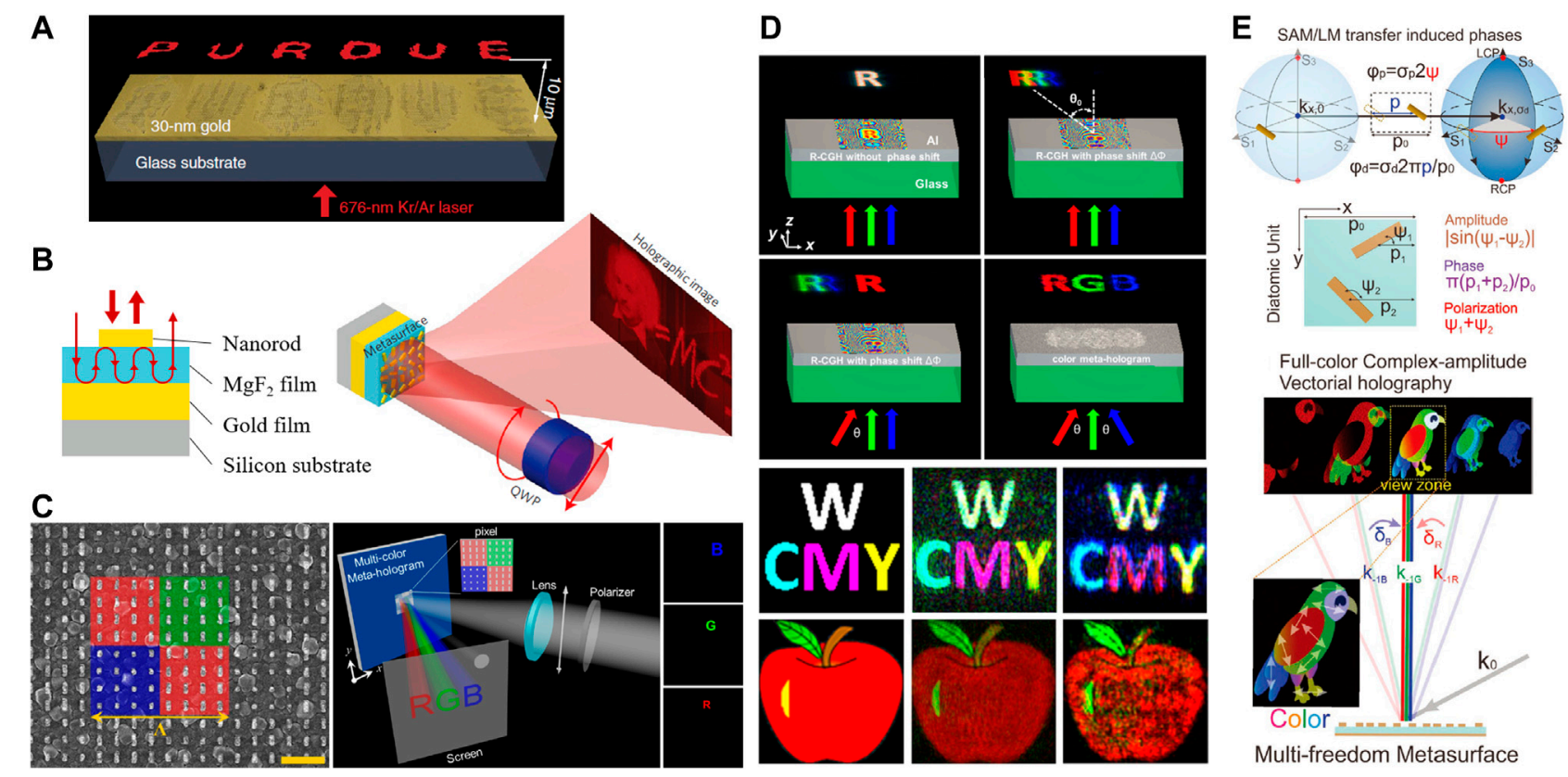

FIGURE 4 | (A) Complementary V-shaped metallic grooves produce a single-color holographic image of the word "PURDUE" [113]. (B) Metasurface hologram with Metal-Insulator-Metal (MIM) structure design for improving the efficiency and shows an Einstein's portrait [193]. (C) Metasurface with super meta-atoms composed of multi-resonators designed for RGB three-primary colors and demonstrates three-colors metasurface hologram [60]. (D) Metasurface hologram with off-axis illumination method realizes the decoding of three primary color components, and further generates their secondary superimposed colors (such as cyan, magenta, yellow, and white) [145]. (E) Design principle of the multi-freedom metasurfaces for full-color meta-hologram [29]. Figure reproduced with permission from: (A), ${ }^{\circ}$ 2013, Nature Publishing Group (NPG); (B), ${ }^{\circledR}$ 2012, Nature Publishing Group (NPG); (C), ${ }^{\circledR}$ 2015, American Chemical Society (ACS); (D), ${ }^{\circledR}$ 2016, American Chemical Society (ACS); (E), ${ }^{\circ}$ 2020, Wiley.

Further on, via modulation of the intensity and phase of RGB (red, green, and blue) laser, (also called color mixing), three-color metasurface holograms can evolve into a full-color hologram. Wan et al. and Li et al. used the off-axis illumination method, that is using three beams of laser (red, green, and blue) to illuminate the metasurface with different tilted incident angles, thus decode three primary color components, and can further generate their secondary superimposed colors (such as cyan, magenta, yellow, and white) $[90,145]$, as shown in Figure 4D. The full-color metasurface holograms were demonstrated by using dielectric metasurfaces, such as silicon nanoblocks [11, 147], silicon nanodisks [188], titanium dioxide $\left(\mathrm{TiO}_{2}\right)$ nanodisks [161] and even graphene oxides [91]. In a very recent work, multi-freedom metasurfaces were proposed, which can modulate phase, amplitude, and polarization simultaneously and independently. Li's group combined the frequency-independent $\mathrm{PB}$ phase and detour phase and realized a complex-amplitude vectorial hologram at various wavelengths, thus a full-color metahologram was demonstrated, as shown in Figure 4E [29].

\section{Metasurface Based Invisible Cloak}

A cloak is a device with the ability to render objects invisible to incoming waves. Transformation optics and metamaterials are offering powerful tools for the development of cloaking devices $[17,81,142]$. Though the concept of the invisible cloak was successfully demonstrated with metamaterials,in practical aspects, development of 3-dimensional cloak with 3D metamaterials is extremely difficult. An alternative approach was first introduced with waveguide based cloaking and it played an important crucial role over a "quasi-conformal mapping technique" where the cloak concealed an object by restoring a wavefront as it was being reflected from a flat surface. This concept was defined as "carpet cloaking" and was demonstrated accordingly [38]. The realization of ultra-thin carpet cloaking was feasible due to the ultra-thin engineered metasurface based structure. Metasurfaces provided an efficient way of designing three-dimensional arbitrary shaped carpet cloaks with ultrathin thicknesses and low loss. The multilayers structure of metasurface was proposed to offer the broadband performance of the cloak [148]. Metallic resonator with discontinuous phase was fabricated over a polyimide substrate. An excellent wavefront manipulation was demonstrated and it was employed over a surface with a triangular bump [158]. The schematic representation of the metasurface carpet clock was shown in Figure 5A. The cloaking layer was fabricated with a standard lithography process, and low loss flexible polyimide layer was used as the spacer medium. Terahertz time-domain spectroscopy with an angular scanning system was used for terahertz cloak demonstration.

The dispersion of materials is always the main challenge in optical components for broadband carpet cloaking. A novel metasurface was proposed recently for carpet cloaking with linear polarization at visible wavelengths from 650 to $800 \mathrm{~nm}$. 

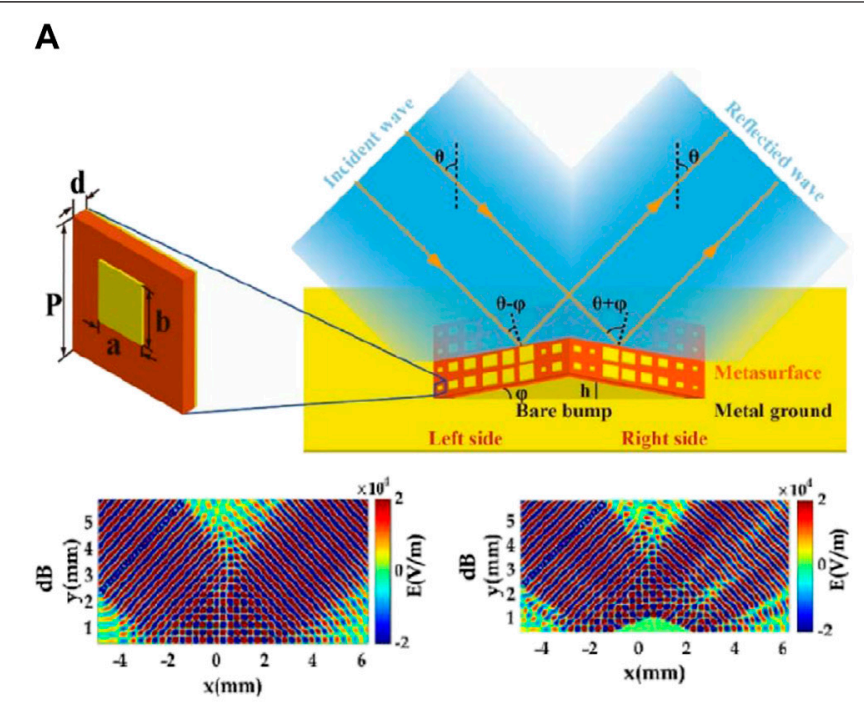

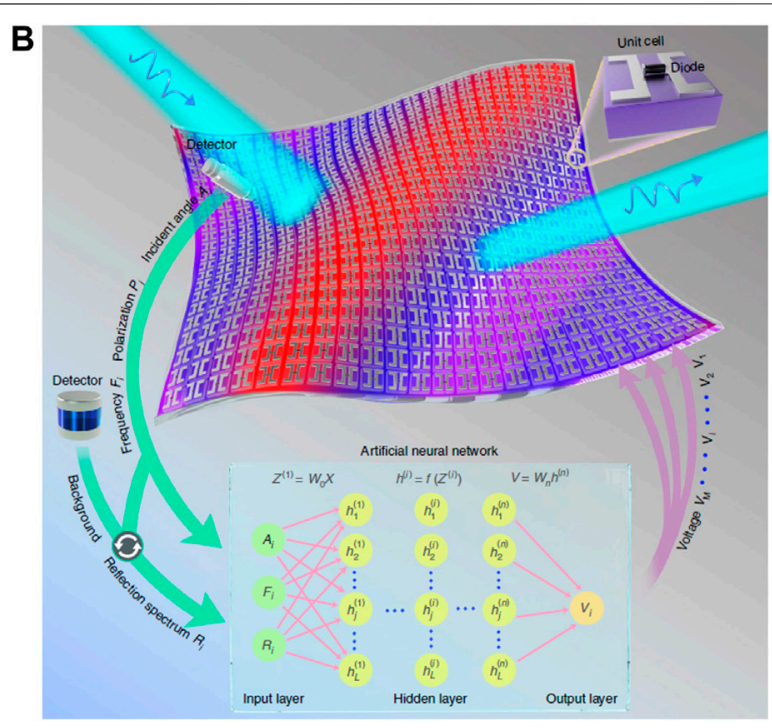

FIGURE 5 | (A)Schematic representation of the metasurface based carpet cloak [158]. (B) Deep-learning-enabled self-adaptive metasurface cloak [126]. Figure reproduced with permission from: (A), ${ }^{\odot}$ 2017, Optical Society of America (OSA); (B), ${ }^{\circledR}$ 2020, Nature Publishing Group (NPG).

In this proposed structure, a thin slot of waveguide based metasurface was presented [50]. Very recently, a metasurface featuring machine learning algorithm was used as an adaptive cloaking system. This work shows the development of nextgeneration invisible cloak with artificial intelligence. An effective tunable metasurface loaded with varactor diodes was used for the fabrication of invisible cloak in the microwave range, as shown in Figure 5B. The metasurface cloak can respond swiftly, on a millisecond timescale with a change in incident wave and surrounding medium by a pre-trained artificial neural network which was a fundamental building block of deep learning [126].

\section{A TREND: FROM SLAB METASURFACE TO FLEXIBLE METASURFACE}

The metasurfaces are required to operate in many other different surfaces apart from large planar wafers for the application of various practical requirements, as well as cooperating with different devices. Thus, the realization of flexible (or soft) metasurface becomes one of the emerging topics nowadays. Soft Metasurfaces are normally being fabricated with metal, metal-dielectric structures, or only dielectric materials deposited over a flexible substrate [12, 22, 69], which are now considered to be the competitive platform for controlling the optical properties of the output light with mechanical force [20]. Meta-structures recently are being developed over flexible polymer [66], 2D materials [61, 99], or carbon nanofilms [138]. To expand the potential application range of flexible metasurface, our group fabricated metastructures on low-cost aluminum foils, which were widely used in food packaging [54]. Numerous modern-day engineering and scientific applications were being carried out over the curvature surface and non-planar surface where the planar sensor/device was outperformed due to the necessary efficiency [66]. Flexible metasurfaces were realized on low surface energy polymers, like polydimethylsiloxane (PDMS). PDMS can adhere to all surfaces in a conformal manner onto curved surfaces, epidermis, and packaging materials [67, 181]. Figure 6A represents the schematic representation of flexible metasurface with PDMS polymer. In this particular case, an amorphous Silicon nano post and a thin layer of $\mathrm{Al}_{2} \mathrm{O}_{3}$ were embedded in a low index flexible PDMS substrate. The thin dielectric metasurface layer conformed to the flexible substrate can modulate the optical response of the flexible substrate.

Soft-metasurface with a randomized phase distribution was employed for diffuse terahertz wave scattering. The distinctly patterned gold layer was deposited over the polyimide substrate for the fabrication of the structure [185]. Figure $6 \mathbf{B}$ represents the microscopic view of the sample as well as its reflection amplitudes with different modes. The energy of surface plasmon polarition of Bloch wave was tuned by flexible meta structures. The tunable plasmonic response was achieved with two layers of gold nanoribbon arrays [99]. The performance of graphene nanoribbon arrays in soft metasurface based plasmonic structures was also investigated in a detailed manner [153]. Randomly distributed meta elements over the polyimide thin films were employed to develop low scattering surface for possible EM stealth technology [187].

Modern and advanced micro and nanofabrication technologies are the basic fabrication tools that are being used for the development of soft and flexible metamaterials [144]. Photolithography, electron-beam lithography, and focused-ionbeam lithography are the most commonly used lithography for patterning nanostructures over the metasurfaces [49, 140, 174]. The resonance pattern over a flexible substrate can be accomplished with electron beam lithography [176], focused 


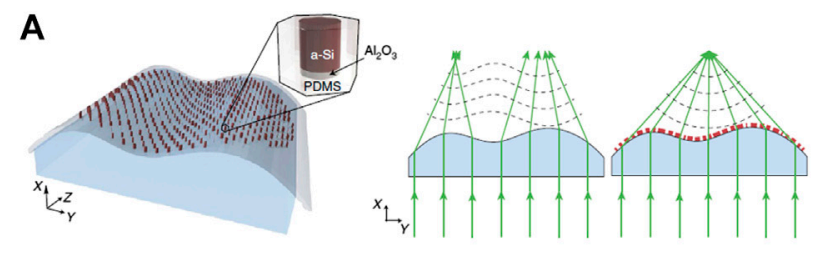

B
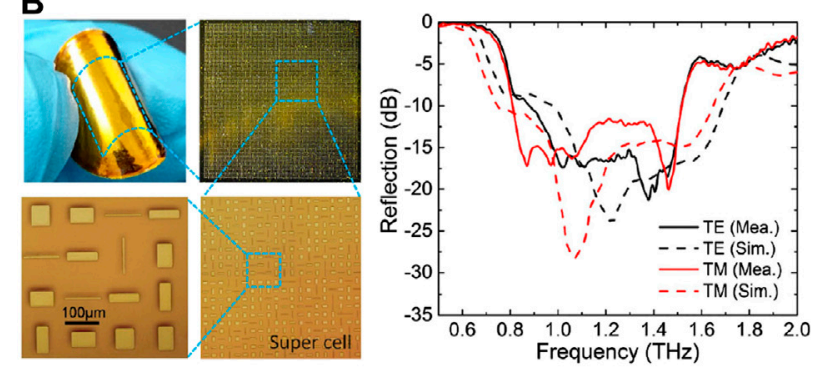

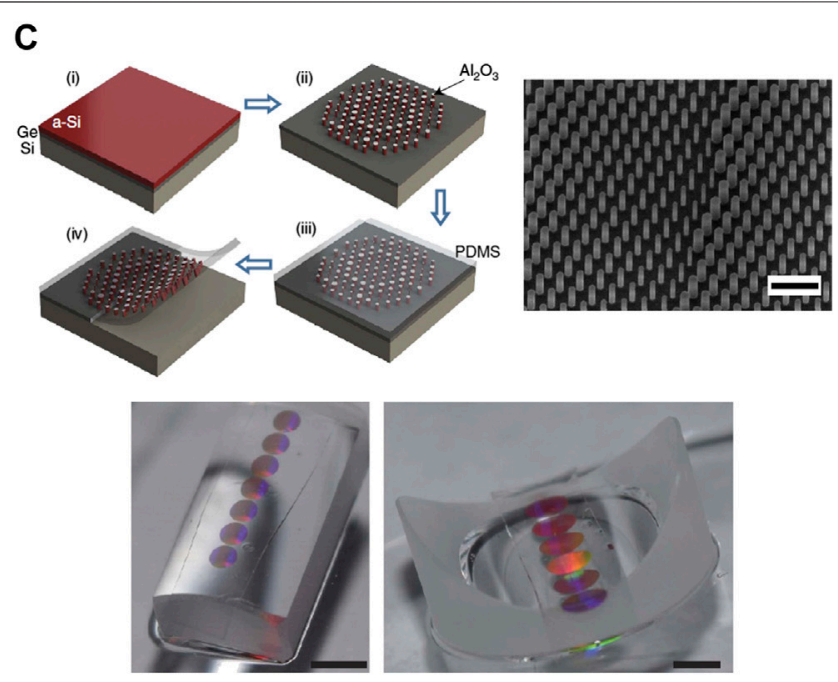

FIGURE 6 | (A) Soft metasurface conformed to the surface of a transparent object with arbitrary geometry to re-shape the wavefront and realize the focusing of transmissive electromagnetic wave [66]. (B) Microscopic pictures of the soft metasurface and its reflection spectrum of transverse electric (TE) and transverse magnetic (TM) modes of the electromagnetic waves within THz frequency domain [185]. (C) Process of developing the soft metasurface via transfer printing [66]. Figure reproduced with permission from: (A)-(C), ${ }^{\circledR}$ 2016, Nature Publishing Group (NPG).

ion beam lithography [40, 113], plasmonic lithography [101], probe scanning lithography [62], laser writing lithography [44, 186] and plasmonic lithography [101]. Nanostencil lithography $[105,149]$ has enabled nanopatterning on a wider range of flexible nonplanar substrates with a simple fabrication process. The softness of the photonics device can be assessed by "mechanical flexibility". Flexibility is normally measured with the maximum possible deformation without any damage in the structure. Yield strength can measure mechanical flexibility and it is defined as maximum allowed strain with deformation of the photonic device. The minimization of the strain of the photonic device layer with deformation force is the key parameter for the fabrication. Minimization of strain can be attained by adopting the so-called "neutral plane" configuration. At the neutral plane, the strain response of the structure vanishes [55].

The transfer of resonance pattern over the flexible substrates needs to be released from the hard substrate which seems to be a delicate part in the designing $[45,66,84]$. Transfer of the metasurface layer over the flexible substrate can be done by transfer printing (or called stamp printing). Two mostly used processes are a) direct flip transfer and b) stamp assisted transfer. Monolithic patterning of soft photonic devices is used often as they provide ease of fabrication, improved yield, and large area patterning capability. The encapsulated devices are peeled off from the rigid handler to complete the fabrication process of the soft photonic devices, as shown in Figure 6C. The sacrificial layer used for successful detachment of flexible metasurface from a rigid substrate. A diluted ammonia solution was used to remove the sacrificial layer from the fabricated structure. Similarly, Ambhire et al. also proposed "Pattern and Peel" method, using PDMS as the sacrificial layer, to fabricate the metasurface on an elastomeric substrate (PDMS) [3]. What's more, they also showed reconfiguration or tunability of the resonance by stretching PDMS layer, and thus realized a kind of reconfigurable metasurface, which will be covered in detailed in next section with various types.

\section{RECONFIGURABLE METASURFACE: AN ENCOURAGING IMPROVEMENT FROM STATIC}

The optical properties of the metasurface are related to engineered structure. Size, shape, and the inter meta-atomic distances as well as the optical parameters of materials control and dictate the optical characteristics of the metasurface. However, traditional metasurfaces are difficult to be tuned after being fabricated, which considerably limit the degree of freedom for full-wave controls. As such, it is highly desirable to realize dynamic metasurfaces, whose functionalities can be actively tunable with controlled external factors. Recent few years, there have been some impressive works published. In this part, the reconfigurable metasurface is being discussed in detail.

\section{Mechanical Force in Soft Metasurface}

Geometric arrangements of resonant building blocks with a fixed material composition drive the performance of the metasurface. The performance of metasurface can be tuned by varying the shape, size, and spatially arrangements of the constituent metaatoms. Mechanical force is the main influence on the tuning of the optical properties of the tunable metasurface. Novel aspects of manipulating light could be achieved with the ability to bend, stretch, and roll of the device on flexible substrates and which promises a new wave of device designs and functionalities [69, $144,174]$. 
Unique and typical characteristics that are being found in metasurface through mechanical deformation is mostly due to the flexible substrates $[69,130,144]$. PDMS, a silicon-based organic polymer is being widely used as an elastomeric substrate due to its low optical loss and excellent elasticity during reversible deformation. Transmittivity of flexible metasurface was demonstrated in detail, and an excellent control over the optical properties by mechanical tuning was shown. Figure 7A depicts that the metasurface exhibits remarkable resonance shifts with mechanical tuning [45]. The interparticle distance was used to modulate the optical properties of metasurface with mechanical stretching. The gap between successive resonators was found to be varied from 10 to $140 \mathrm{~nm}$ with $0-100 \%$ applied strain over PDMS substrates [97]. The mechanical flexibility of engineered metasurface was explored in tuning the focal length of meta-lens. All-dielectric material metalens was demonstrated where amorphous silicon post was developed over the PDMS substrate [68]. The mechanically tunable lens in visible wavelength was also demonstrated with significant efficiency in controlling the focal length. The lattice constant of $\mathrm{Au}$ nanorod was tuned which is fabricated over PDMS substrate [33]. Polarization of the incident wave can be tuned with chirality of the metasurface, and nanostructures with chiral geometries exhibit strong polarization rotation. Plasmonic nano colloids with rotary optical activity were developed with twisted PDMS substrate and multilayers of metal nanoparticles [76].

\section{From Microelectromechanical Systems to Programmable Metasurface}

Microelectromechanical systems (MEMS) offers a method to realize the structural reconfiguration of the metasurface, thus modifying the electromagnetic response. Metasurface based midIR flat lens was developed for active beam steering [128]. The design of microcantilever arrays in metasurface allowed the structure can be tuned between the "touch" and "separate" by the electrostatic force $[28,120,190]$. Figure 7B presents a MEMS metasurface with micro-cantilever, which realizes structural tunability with electricity. There were significant shifts of resonance wavelength as one end of unfixed open ring resonators gradually warps. Discrete structures, which stood on substrates with different properties, offered the possibility of modification [182]. As shown in Figure 7C, the metasurface composes of two types of slabs. One kind of slabs patterned on a rigid substrate and the other one was suspended, which can be shifted by a micromachined actuator. However, recently explored MEMS metasurfaces generally work in the Terahertz band, microwave regime, and range with longer wavelengths, which have a large size with a dimension of tens of micrometers or even larger. For those more compact meta-structures working in a higher-frequency region, one needs to explore special sophisticated methods, such as Nanoelectromechanical systems technology. Further on, blending self-feedback and programming algorithms into the metasurface, programmable metasurface can be achieved. More recently, Cui et al. utilized the diodes and fieldprogrammable gate array, which embedded into the metasurface, to store a bulk of coding sequences as well as corresponding electromagnetic responses, and realized a smart metasurface [103]. Figure 7D presents the appearance of this reprogrammable metasurface. Adaptive single-beam, multibeam manipulation, and other dynamic reactions were demonstrated successfully. In recent years, with the rise of artificial intelligence, concepts such as machine learning have emerged in many fields. With the aid of machine learning, reprogrammable metasurface will also be more intelligent [87]. This is undoubtedly a promising direction in the field of metasurface research.

\section{Structures With Tunable Materials}

The reconfigurable metasurface mentioned above is achieved by changing the structure (shape, arrangement). Utilizing those tunable materials, as a part of the structure design, can also be a strategy to realize the modification of metasurface. Phasechange materials (PCMs), such as the GeSbTe (short for GST) alloys, suffer from significant optical parameters variation between the amorphous and crystalline states, and thus recently being used to modify the optical properties of metasurface $[15,25,30]$. Galarreta et al. embedded a thin layer of GST into silicon nanodisks and realized the modification of resonance [129]. In addition to the amorphous and crystalline states, stepwise intermediates states were also demonstrated [154].

Graphene has received a lot of attention since its discovery. It can also be used to achieve reconfigurable metasurfaces due to its adjustable band gap and excellent optical properties. A monolayer graphene sheet was patterned on the silicon photonic crystal-like substrate and realized tunable induced transparency via the electrical method [23]. Fan et al. used a pump beam to excite the graphene and enhanced the initially weak magnetic resonances significantly [35]. Similarly, giving stimuli according to other embedded materials sensitive to a thermal, electric, or optical stimulus can also get a tunable metasurface. For example, silicon illuminated with infrared pump beam can produce photocurrent and tune the resonance of metallic metasurface [42]. Recently, ferromagnetic particles were mixed in the 3D-printed soft materials and realized the actively controlling of the shape under applied magnetic fields [77], which can be also referred to in the metasurface design.

\section{Fluidic Unit Cell Metasurface}

Microfluidic technology [13, 106] enables people to control liquids in various fields within micro-/nanoscale, which matches the structural dimensions of the metasurface well. Recent few years, the combination of microfluidic and metasurface is emerging gradually [184]. Injecting liquid into a sub-wavelength periodic channel structure, i.e., "liquid cell metasurface", and using the fluidity of the liquid or liquid material, personalized micro-structure units can be created to customize the metasurface optical response. Besides, more applications are required to be completed in a liquid environment, such as bio-sensing, bio-detection, and bioimaging. Different optical responses can be obtained by injecting different solutions into the water-cell metasurface microstructure. Most water-cell metasurfaces are used as 

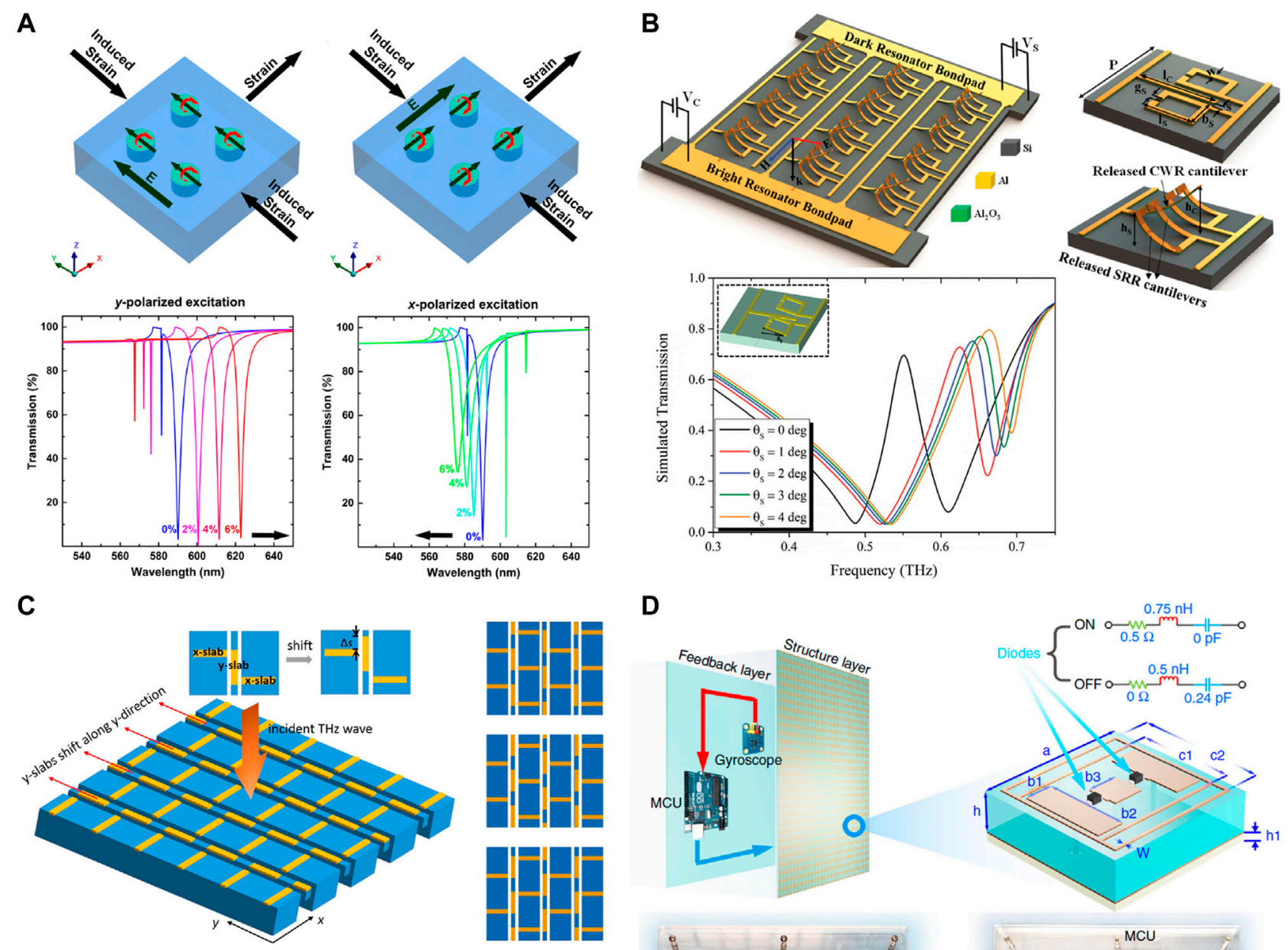

D
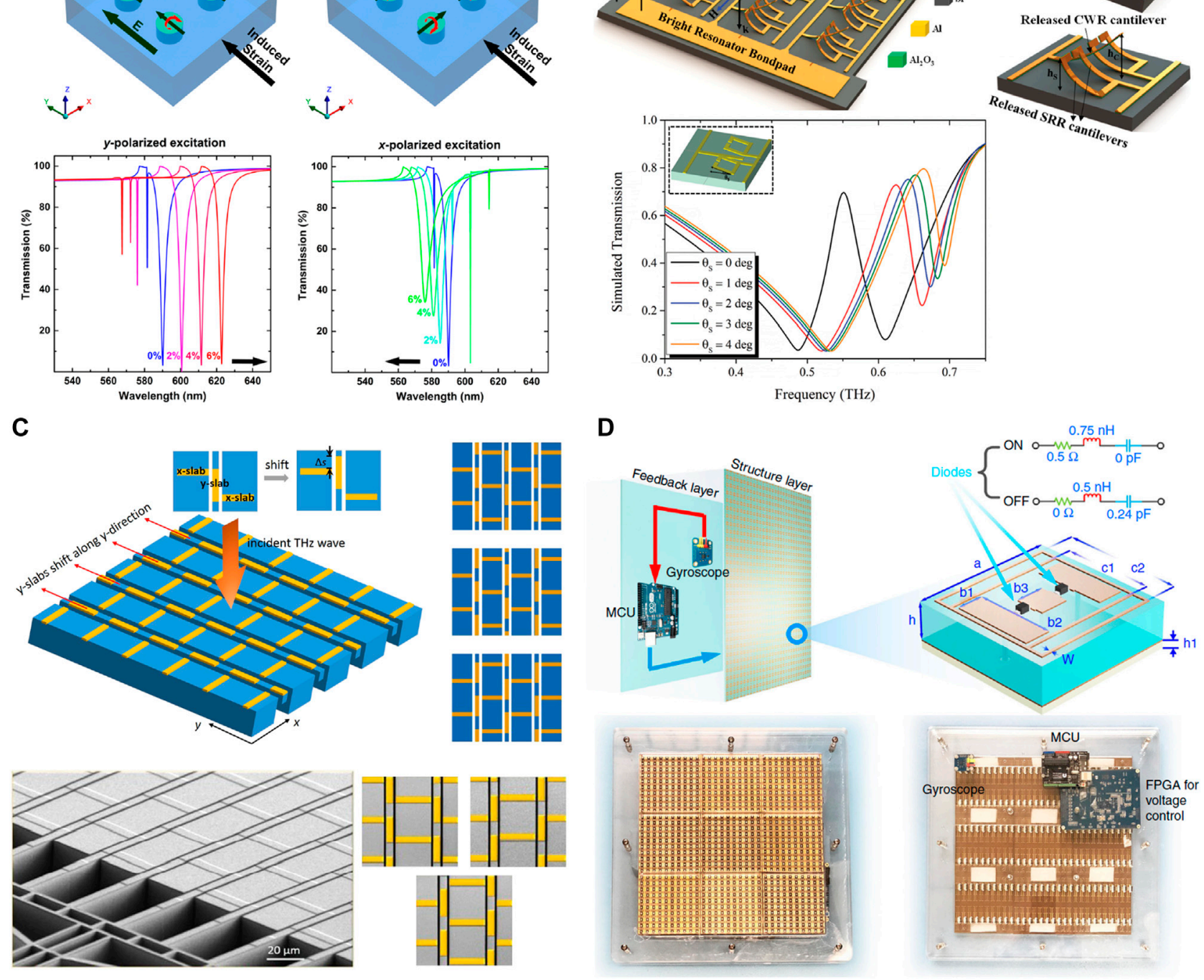

FIGURE 7 | (A) Stretch the flexible metasurface and realize remarkable resonance shifts [45]. (B) MEMS metasurface with cantilevers allow structures can be tuned via electricity voltage. There are significant shifts of resonance wavelength as one end of unfixed open ring resonators gradually warps [120]. (C) The reconfigurable metasurface with suspended MEMS structure design and its three reconfigurable states [182]. (D) Schematic diagram and actual appearance of programmable metasurface [103]. Figure reproduced with permission from: (A), ${ }^{\odot}$ 2016, American Chemical Society (ACS); (B), ${ }^{\circ} 2016$, Wiley; (C), ${ }^{\odot}$ 2017, Nature Publishing Group (NPG); (D), ${ }^{\odot}$ 2019, Nature Publishing Group (NPG).

sensors to detect incoming samples. For example, a THz system that combines highly sensitive and non-invasive detection of biological samples with metasurfaces and microfluidics was developed recently [59], and an ultra-sensitive fluid sensor capable of determining the type and concentration of chemical reagent with high accuracy was demonstrated successfully [1]. All of these have laid the foundation for accurate detection in the future medical chemistry and other fields. While the liquid-metal is a substance with fluidity, metallicity, and thermal expansion characteristics. Mercury $(\mathrm{Hg})$ was first used in the metasurface molecules in the microwave band to regulate the propagation of waves, as shown in Figure 8A [70]. However, $\mathrm{Hg}$ is highly toxic and thus be replaced by some alloys such as eutectic galliumindium (EGaIn). In Figure 8B, by injecting EGaIn into PDMS mold, the first $\mathrm{THz}$ liquid-metal-cell metasurface was born [150, 151]. Later, the metasurface composed of small tubes infused with liquid-metal EGaIn and copper wire pairs completed the tuning of switching the electromagnetically induced transparency (EIT)like response [164]. The liquid-metal alloy EGaIn was injected into the microfluidic channel on the PMMA substrate, and the 

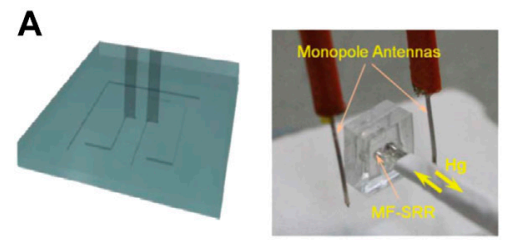

B

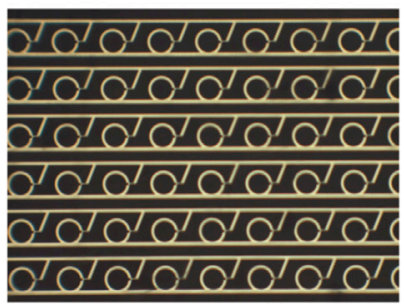

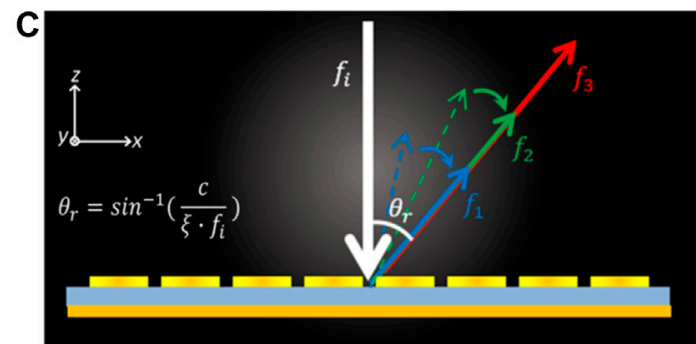
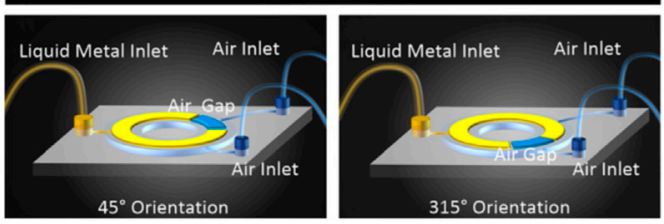

FIGURE 8 | Structures of liquid-metal-cell metamaterials. (A) Reconfigurable electromagnetic metamaterials using Hg in metamolecular [70]. (B) Reconfigurable THz metamaterial device with EGaln in microchannels [150]. (C) Individually modulating ring-resonator-array metamaterial using liquid-metal Galinstan [166]. Figure reproduced with permission from: (A), ${ }^{\odot}$ 2009, AIP Publishing; (B), ${ }^{\odot}$ 2014, Optical Society of America (OSA); (C), ${ }^{\odot}$ 2017, AIP Publishing.

shear of the resonance frequency from 10.96 to $10.61 \mathrm{GHz}$ was completed while maintaining the absorption rate up to $98 \%$ [95]. As for more precise reconfiguration, a metasurface for periodically distributed ring resonators was designed as shown in Figure 8C, which can modulated the shape of the liquid-metal Galinstan, a gallium indium tin alloy, in each ring resonator individually, and then dynamically adjusted the light reflection [166]. According to the thermal expansion of liquid-metal mercury, different broadband $\mathrm{THz}$ metasurface absorbers were proposed and can be customized at different temperatures [180].

\section{Metasurface in Fluid-Background}

The fluid-background metasurface is a solid-liquid mixed metasurface. The developed metasurface is immersed in water, alcohol, liquid crystal (LC), or even a solution of suspended nanoparticles, to achieve the corresponding purpose of induced tuning optic waves. Water is the most abundant liquid resource on earth. Because it is cheap and non-toxic, and can be miscible with a large number of liquids, it is a very common liquid surrounding material and different structures were proposed over the years [32, 56]. Ethanol [75], glucose solution [195], sucrose solution [114], etc. are also often used as background material. By adjusting the concentration of the solution covering the solid metasurface, the refractive index of the solution is changed, and then the dielectric constant is changed, and finally, the optical response is adjusted [114]. In recent years, LC has attracted much attention as a promising background material of metasurface. It has both crystallinity and fluidity at room temperature. By changing the external conditions, the orientation of the LC molecules can be redirected to achieve a tunable optical response. The excellent characteristics of LC make it applicable in many fields. Liu's group has designed several reconfigurable chiral metasurface absorbers by using a LC layer as an insulator layer. In one structure, the circular dichroism of the incident light will be modulated with the different alignments of the LC molecules, which could achieve the effect of reversing circular dichroism [172]. Other plasmonic LC reconfigurable chiral metasurfaces of sandwich-structure, as shown in Figure 9A, were simulated to spin-selectively absorb CP light at multiband frequencies [160] and efficiently [171]. Recently, $\mathrm{THz}$ beam was manipulated by programmable LC background metasurfaces, as shown in Figure 9B. In this article, the "0" and "1" encoding technology was used to switch the state of different arrays to redirect the LC molecules in it, thereby changing the refractive index of different regions, and finally realize the dynamic manipulation of the $\mathrm{THz}$ wave, and its maximum deflection angle reached $31.2 \%$ [159]. Similarly, a digital metasurface device for light projection display was demonstrated with each pixel is electrically reconfigurable [82]. A LC plasma metasurface was also developed to display electronically adjustable color labels [135]. Metasurfaces with LC as the surrounding material was used as all-optical switches [8] and switches of controlling the generation of second harmonics of metasurface [127]. In terms of controlling electromagnetic radiation, the LC-based metasurface was also designed to successfully achieve dynamic thermal camouflage, as shown in Figure 9C, which provides a new development direction for the future thermal metasurface with emissivity engineering [100]. Nanoparticle solutions can also be used a background materials as same as LC. The orientation of the nanoparticles suspended in the solution can be redirected and rearranged by tuning the external factors, and thus the light will be controlled [48].

\section{A NEW PLATFORM: METASURFACE ON FIBER}

After the demonstration of the powerful capability of electromagnetic wave manipulation by metasurface, it has been successfully applied to optical fibers, bringing immense advantages over its domain of applications. Thus amalgamation of metasurface and optical fibers provide a powerful platform for the development of novel optical components. With the emergence of "Lab of Fiber", micro-, 

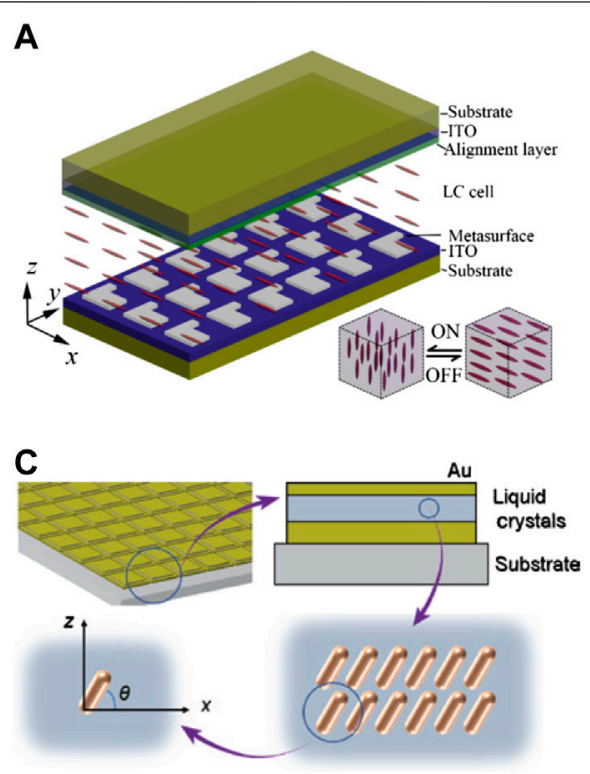

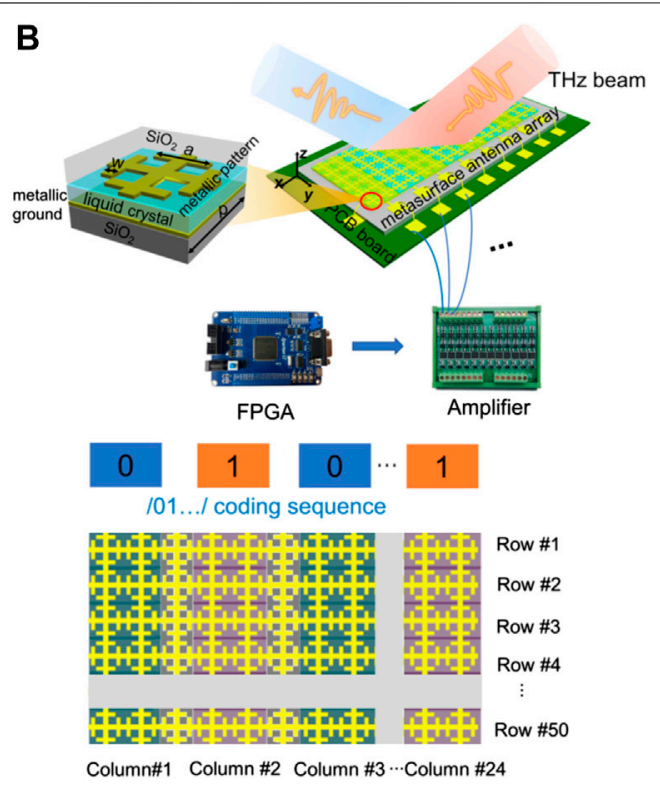

FIGURE 9 | Structures of LC background metamaterials. (A) Reconfigurable spin-selectively multiband absorber based on LC chiral metasurface [160]. (B) Codeable LC metamaterial for controlling THz beam [159]. (C) Metal-LC-Metal platform with dynamic thermal camouflage function [100]. Figure reproduced with permission from: (A), ${ }^{\circledR}$ 2018, Optical Society of America (OSA); (B), ${ }^{\circledR}$ 2020, AlP Publishing; (C), ${ }^{\circ}$ Walter de Gruyter GmbH 2020.

nano-structure, and metasurface are fabricated on/in/around the optical fiber to realize specific functions $[37,125,163,191]$.

Optical fiber devices decorated with micro-structures and functional materials have been studied for decades. Still, the surface decoration based on traditional self-assembly is almost out-of-order and uncontrollable. Thus, the ability to fabricate controllable structures on optical fiber will help the further expansion of the application. However, considering that most of the micro- and nanofabrication methods arising from the microelectronic industry operate on large planar substrates, fabrication of metasurface on the fiber is difficult. Researchers are contributing their wisdom to combine fiber optics with metasurfaces.

One primitive approach is self-assembly lithography. Since the nanospheres float in a regular arrangement at the interface between air and non-polar solvent, which is also called Langmuir-Blodgett procedure [7]. Pisco et al. used selfassembled polystyrene nanospheres as a mask and fabricated round hole periodic structure on the facet of fiber [118], as shown in Figure 10A. Similarly, a polymer solution with water droplets assembled as a close pack array inside was also used. The template was accomplished with evaporation of the water. They realized a round-hole periodic structure on the facet of fiber with the help of the holder [119]. Further on, this kind of regularly arranged nanostructures templates was used as Kaleidoscope shadow masks when illuminated with different incident angles, and thus makes it possible to fabricate more periodic structures [110], as shown in Figure 10B.

However, self-assembly lithography can only satisfy a limited number of simple structures. For more complicated structures, one needs to explore a more competent method for an advanced level of fabrication. Principe et al. utilized a holder to clamp the single-mode fiber and deposited a gold layer on the facet of fiber via electron beam evaporation, subsequently fabricated a rectangular groove array, which covered the entire core regime, with focused-ion-beam nanofabrication processed directly [125]. As shown in Figure 10C, the rectangular grooves with different lengths on the fiber end-facet produced various phase jumps, and the anomalous transmission in optical fiber was firstly demonstrated on this fiber meta-tip. Later, controlled absorption was also demonstrated in the optical fiber system with a similar method, and can be further used in logical operation [163].

This direct lithography method ensures that the shape of the fabricated metasurface structure can meet the requirements to a greater extent as one wishes. However, the resolution of standard UV lithography is over $1 \mu \mathrm{m}$ while the size of the meta atoms on fiber end-facet is approximately $\sim 100 \mathrm{~nm}$ or even smaller. E-beam lithography and FIB are normally used for the fabrication of the metasurface on fiber. What's more, compared with flat wafers, optical fibers are less compatible and controllable in lithography process. The scalability issue of the fabrication of metasurface are limited by the following factors: 1) The flatness and smoothness of the surface of the fiber tip; 2) The stability of the position of the fiber, as special mount is required to put the fiber vertically below the electron beam, a slight shift of the fiber from its position could affect the designing in a massive manner; 3 ) The size and pattern of the meta-atoms, which also greatly affect the speed and efficiency of the fabrication. It needs to be mentioned that both e-beam lithography and FIB fabrications methods are not industrial grade. So specific optimization processes are required to be followed for each of the specific fabrications. The fabrication of meta atoms over the planar device is a bit easier to optimize 


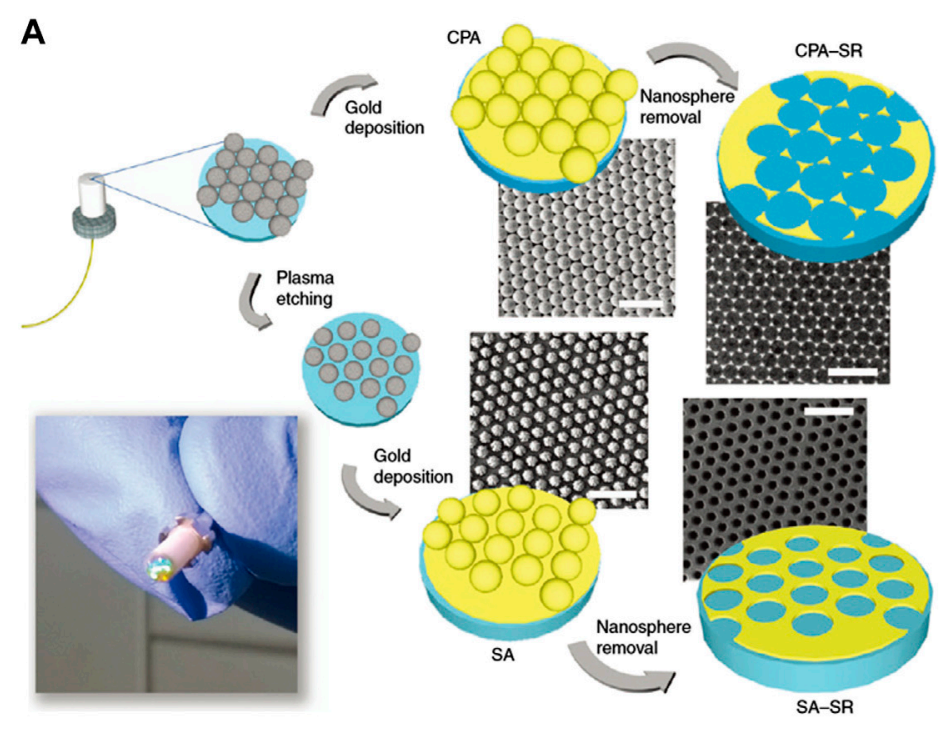

C

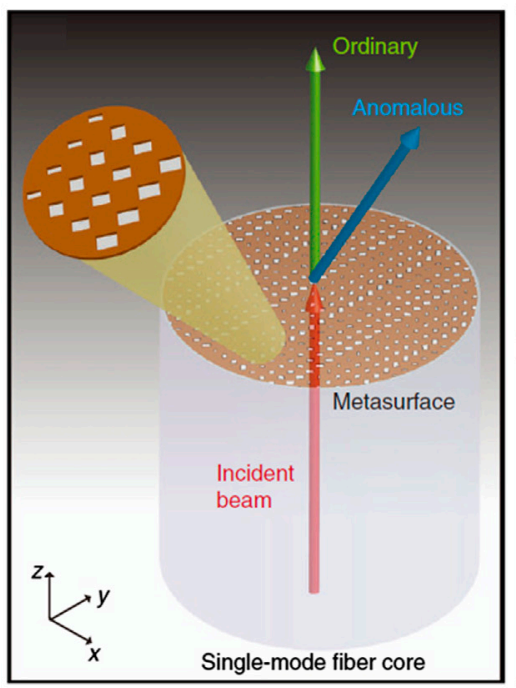

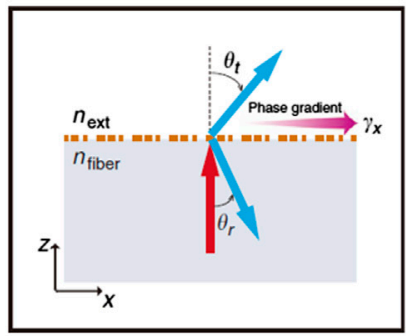

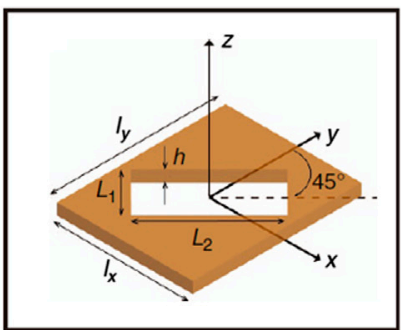

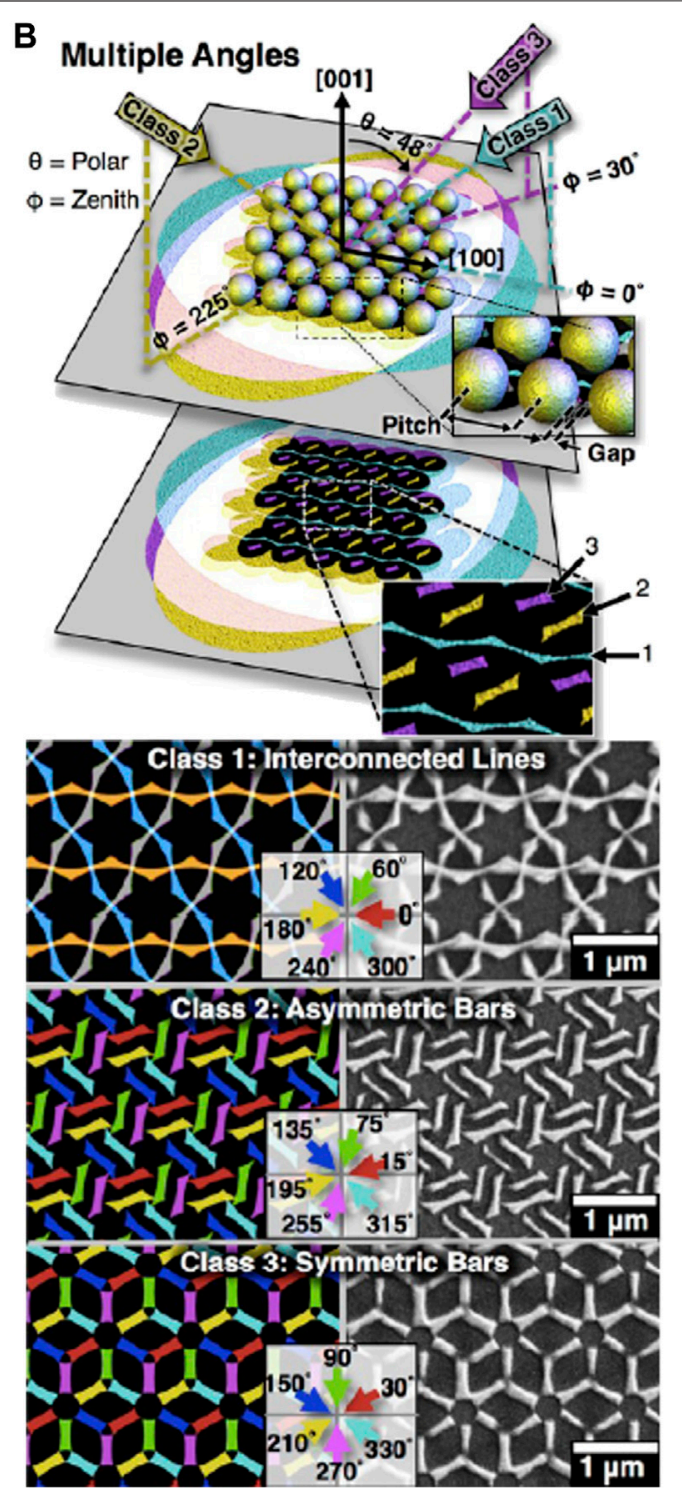

B

FIGURE 10 | (A) Fabrication process of meta-structure on the fiber end-facet via self-assembly lithography [118]. (B) Meta-structures fabricated with Kaleidoscope shadow masks [110]. (C) Anomalous transmission on fiber meta-tip [125]. Figure reproduced with permission from: (A), ${ }^{\odot}$ 2017, Nature Publishing Group (NPG); (B), ${ }^{\odot}$ 2014, American Chemical Society (ACS); (C), ${ }^{\circledR}$ 2017, Nature Publishing Group (NPG).

than that required for optical fiber. As a result, it would be extremely inefficient to make these nano-structures directly on optical fibers.

Recently, transfer of nano-structure from a large wafer onto the fiber end-facet became a potential method to make metasurface on fiber [157]. Juhl et al. utilized UV-curable hybrid polymer to transfer the gold nanoantennas array from silicon wafer to the fiber tip completely, due to the soft and quickly-dry of UV-curable hybrid polymer [65]. Although it still takes a lot of labor and resources to make fine patterns on the wafer in advance, the transfer of the metasurface from the wafer to the fiber is not really difficult. As long as the wafer template is available, mass production of fiber meta-tips may become feasible. In addition, it is also promising to transfer these elaborate structures to the side-surface of the fiber by rolling the fiber and combining with this kind of transcription process.

Nanoinstile lithography, roll to roll printing techniques are used to make the process more appropriate but till today the fabrication of the meta-atoms over the fiber remains a challenge. To date, the metasurface is only developed on the flat end-facet of the fiber, almost no metasurface around the arc surface of the fiber has been demonstrated, let alone those inside of the photonic crystal fiber. In a word, there is still a long way to realize the integration of metasurface into an optical fiber system. 


\section{CONCLUSIONS AND OUTLOOK}

As the latest generation of optical devices, the compactness of metasurface is unmatched by traditional optical devices. Metasurface can perform wavefront regulation at the subwavelength scale and has attracted extensive attention since its adventless than a decade ago. In this review, we have summarized the basic principles of metasurface in the reshaping of electromagnetic waves based on the phase and amplitude modulation, as well as its mainstream applications. Considering metasurface analogues are so attractive due to their amazing optical properties, metasurfaces are combined on another powerful optical device: optical fibers. This part of the explorations was also reviewed in this paper. From here, we can see that the working environment of a metasurface is by no means limited to large flat wafers. It needs to be integrated with other components, work on any surface, and adapt to a variety of environments. Thus, we retrospect that pioneering research works about flexible metasurface and reconfigurable metasurface.

It should be noted that, due to the vastness and rapid development of this field involved as well as our limited cognition and energy, it is impossible for cover all important aspects within this review, such as nonlinear metasurfaces $[19,78$, 80, 170], hyperbolic metasurfaces [46, 64, 88] and so on. Some branches are just mentioned briefly. However, they are immensely important and play a crucial rule in their respective applications but a detailed discussion is out of scope of this particular review article. Before conclusion of this review, we would like to comment on future prospects of metasurface. From a functional device perspective, if the metasurface is only satisfied with the realization of functions on large flat wafers, it is not only unrealistic, but also has no future.

A simple way to make the study of metasurface more practical is to combine it with other more mature fields and functional devices. Due to the author's long working experience in the field of optical fiber, more attention is paid to the combination of this aspect. To date, there are only some simple metasurface diagrams developed on the flat end-facet of the fiber, or the so-called meta-tips. There is almost no metasurface developed around the arc surface of the fiber, let alone the metasurface inside of the photonic crystal fiber. More recently, Steiner and co-workers proposed a 'self-rolled

\section{REFERENCES}

1. Abdulkarim YI, Deng L, Karaaslan M, Altintas O, Awl HN, Muhammadsharif FF, et al. Novel metamaterials-based hypersensitized liquid sensor integrating omega-shaped resonator with microstrip transmission line. Sensors. (2020) 20(3):943. doi:10.3390/s20030943

2. Aieta F, Genevet P, Kats MA, Yu N, Blanchard R, Gaburro Z, et al. Aberrationfree ultrathin flat lenses and axicons at telecom wavelengths based on plasmonic metasurfaces. Nano Lett. (2012) 12(9):4932-6. doi:10.1021/nl302516v

3. Ambhire SC, Palkhivala S, Agrawal A, Gupta A, Rana G, Mehta R, et al. "Pattern and Peel" method for fabricating mechanically tunable terahertz metasurface on an elastomeric substrate. Opt Mater Express. (2018) 8(11):3382-91. doi:10.1364/ ome.8.003382 multilayer metasurfaces' based on templates that can roll themselves up [12]. This method is similar to the Nanoimprint lithography $[47,131,132]$, while implemented on the soft materials. Referring to this approach, metasurface may be developed around the arc surface of the fiber easily. In addition, the microstructure on the fiber is mostly used in the nearinfrared communication band, which greatly limits the size of the microstructure to the sub-micron scale. At present, it can only be achieved by electron beam exposure, which heavily aggravates the difficulty of mass processing. If one could successfully implement the integration of metasurface into an optical fiber system and realize the lab on/around/in fiber, it would be a large step in optical devices. Although there are still more efforts required.

On the other hand, the integration of metasurface with other emerging concepts is also a promising direction. With the rise of artificial intelligence, various concepts such as artificial intelligence algorithms and data mining are expected to make metasurfaces more powerful [87, 103]. More recently, metasurface was utilized in the field of quantum optics, and the quantum behavior of superposition and correlation between homologous multi-photon pairs was experimentally verified [86]. This paper verified the feasibility of high dimensional quantum entanglement chip. We believe that with the joint efforts of many researchers, the research of metasurface will make swift progress, radiate to more fields, and promote the development of science and technology.

\section{AUTHOR CONTRIBUTIONS}

$\mathrm{JH}$ wrote most content of this review article; SB and YL helped JH to finish the whole review; LS supervised the whole work. All the authors reviewed the manuscript.

\section{FUNDING}

This work was supported in part by the Post-doctoral research fund and Start-up Funding of Southern University of Science and Technology under Grant No. Y01236128.

4. Arbabi A, Horie Y, Bagheri M, Faraon A. Dielectric metasurfaces for complete control of phase and polarization with subwavelength spatial resolution and high transmission. Nat Nanotechnol. (2015) 10(11):937-43. doi:10.1038/nnano. 2015.186

5. Arbabi A, Horie Y, Ball AJ, Bagheri M, Faraon A. Subwavelength-thick lenses with high numerical apertures and large efficiency based on high-contrast transmitarrays. Nat Commun. (2015) 6:7069. doi:10.1038/ncomms8069

6. Arbabi E, Arbabi A, Kamali SM, Horie Y, Faraji-Dana M, Faraon A. MEMStunable dielectric metasurface lens. Nat Commun. (2018) 9(1):812. doi:10.1038/ s41467-018-03155-6

7. Ariga K, Yamauchi Y, Mori T, Hill JP. 25th anniversary article: what can be done with the Langmuir-Blodgett method? Recent developments and its critical role in materials science. Adv Mater. (2013) 25(45):6477-512. doi:10.1002/adma. 201302283 
8. Atorf B, Muhlenbernd H, Zentgraf T, Kitzerow H. All-optical switching of a dye-doped liquid crystal plasmonic metasurface. Opt Express. (2020) 28(6): 8898-908. doi:10.1364/OE.383877

9. Avayu O, Almeida E, Prior Y, Ellenbogen T. Composite functional metasurfaces for multispectral achromatic optics. Nat Commun. (2017) 8:14992. doi:10.1038/ ncomms14992

10. Bao Y, Ni J, Qiu CW. A minimalist single-layer metasurface for arbitrary and full control of vector vortex beams. Adv Mater. (2020) 32(6):e1905659. doi:10. 1002/adma.201905659

11. Bao Y, Yu Y, Xu H, Guo C, Li J, Sun S, et al. Full-colour nanoprint-hologram synchronous metasurface with arbitrary hue-saturation-brightness control. Light Sci Appl. (2019) 8:95. doi:10.1038/s41377-019-0206-2

12. Bermúdez-Ureña E, Steiner U. Self-rolled multilayer metasurfaces. ACS Photonics. (2019) 6(9):2198-204. doi:10.1021/acsphotonics.9b00816

13. Bhatia SN, Ingber DE. Microfluidic organs-on-chips. Nat Biotechnol. (2014) 32(8):760-72. doi:10.1038/nbt.2989

14. Cai BG, Li YB, Jiang WX, Cheng Q, Cui TJ. Generation of spatial Bessel beams using holographic metasurface. Opt Express. (2015) 23(6):7593-601. doi:10. 1364/OE.23.007593

15. Cao T, Cao Y, Fang L. Reconfigurable parity-time symmetry transition in phase change metamaterials. Nanoscale. (2019) 11(34):15828-35. doi:10.1039/ c9nr01828j

16. Chen BH, Wu PC, Su VC, Lai YC, Chu CH, Lee IC, et al. GaN metalens for pixel-level full-color routing at visible light. Nano Lett. (2017) 17(10):6345-52. doi:10.1021/acs.nanolett.7b03135

17. Chen H, Zheng B, Shen L, Wang H, Zhang X, Zheludev NI, et al. Ray-optics cloaking devices for large objects in incoherent natural light. Nat Commun. (2013) 4:2652. doi:10.1038/ncomms3652

18. Chen L, Ma Q, Jing HB, Cui HY, Liu Y, Cui TJ. Space-energy digital-coding metasurface based on an active amplifier. Phys Rev Appl. (2019) 11(5):054051. doi:10.1103/PhysRevApplied.11.054051

19. Chen S, Reineke B, Li G, Zentgraf T, Zhang S. Strong nonlinear optical activity induced by lattice surface modes on plasmonic metasurface. Nano Lett. (2019) 19(9):6278-83. doi:10.1021/acs.nanolett.9b02417

20. Chen WT, Zhu AY, Capasso F. Flat optics with dispersion-engineered metasurfaces. Nat Revi Mater. (2020) 5:604-20. doi:10.1038/s41578-020-0203-3

21. Chen X, Huang L, Muhlenbernd H, Li G, Bai B, Tan Q, et al. Dual-polarity plasmonic metalens for visible light. Nat Commun. (2012) 3:1198. doi:10.1038/ ncomms 2207

22. Chen Y, Ai B, Wong ZJ. Soft optical metamaterials. Nano Converg. (2020b) 7(1):18. doi:10.1186/s40580-020-00226-7

23. Chen ZH, Tao J, Gu JH, Li J, Hu D, Tan QL, et al. Tunable metamaterialinduced transparency with gate-controlled on-chip graphene metasurface. $O p t$ Express. (2016) 24(25):29216-25. doi:10.1364/OE.24.029216

24. Cheng Q, Ma M, Yu D, Shen Z, Xie J, Wang J, et al. Broadband achromatic metalens in terahertz regime. Sci Bull. (2019) 64(20):1525-31. doi:10.1016/j. scib.2019.08.004

25. Choi C, Lee SY, Mun SE, Lee GY, Sung J, Yun H, et al. Metasurface with nanostructured $\mathrm{Ge} 2 \mathrm{Sb} 2 \mathrm{Te} 5$ as a platform for broadband-operating wavefront switch. Adv Opt Mater. (2019) 7(12):1900171. doi:10.1002/adom.201900171

26. Choudhury S, Guler U, Shaltout A, Shalaev VM, Kildishev AV, Boltasseva A. Pancharatnam-Berry phase manipulating metasurface for visible color hologram based on low loss silver thin film. Adv Opt Mater. (2017) 5(10): 1700196. doi:10.1002/adom.201700196

27. Chu H, Qi J, Qiu J. An efficiently-designed wideband single-metalens with high-efficiency and wide-angle focusing for passive millimeter-wave focal plane array imaging. Opt Express. (2020) 28(3):3823-34. doi:10.1364/OE. 385498

28. Cong L, Pitchappa P, Lee C, Singh R. Active phase transition via loss engineering in a terahertz MEMS metamaterial. Adv Mater. (2017) 29(26): 1700733. doi:10.1002/adma.201700733

29. Deng ZL, Jin M, Ye X, Wang S, Shi T, Deng J, et al. Full-color complexamplitude vectorial holograms based on multi-freedom metasurfaces. $A d v$ Funct Mater. (2020) 30(21):1910610. doi:10.1002/adfm.201910610

30. Ding F, Yang Y, Bozhevolnyi SI. Dynamic metasurfaces using phase-change chalcogenides. Adv Opt Mater. (2019) 7(14):1801709. doi:10.1002/adom. 201801709
31. Ding X, Monticone F, Zhang K, Zhang L, Gao D, Burokur SN, et al. Ultrathin pancharatnam-berry metasurface with maximal cross-polarization efficiency. Adv Mater. (2015) 27(7):1195-200. doi:10.1002/adma.201405047

32. Ebrahimi A, Withayachumnankul W, Al-Sarawi S, Abbott D. High-sensitivity metamaterial-inspired sensor for microfluidic dielectric characterization. IEEE Sensor J. (2014) 14(5):1345-51. doi:10.1109/jsen.2013.2295312

33. Ee HS, Agarwal R. Tunable metasurface and flat optical zoom lens on a stretchable substrate. Nano Lett. (2016) 16(4):2818-23. doi:10.1021/acs. nanolett.6b00618

34. Estakhri NM, Alù A. Recent progress in gradient metasurfaces. JOpt Soc Am B. (2015) 33(2):A21. doi:10.1364/josab.33.000a21

35. Fan Y, Shen N-H, Zhang F, Zhao Q, Wei Z, Zhang P, et al. Photoexcited graphene metasurfaces: significantly enhanced and tunable magnetic resonances. ACS Photonics. (2018) 5(4):1612-8. doi:10.1021/acsphotonics. $8 \mathrm{~b} 00057$

36. Fu YH, Kuznetsov AI, Miroshnichenko AE, Yu YF, Luk'yanchuk B. Directional visible light scattering by silicon nanoparticles. Nat Commun. (2013) 4:1527. doi:10.1038/ncomms2538

37. Galeotti F, Pisco M, Cusano A. Self-assembly on optical fibers: a powerful nanofabrication tool for next generation "lab-on-fiber" optrodes. Nanoscale. (2018) 10(48):22673-700. doi:10.1039/c8nr06002a

38. Gharghi M, Gladden C, Zentgraf T, Liu Y, Yin X, Valentine J, et al. A carpet cloak for visible light. Nano Lett. (2011) 11(7):2825-8. doi:10.1021/nl201189z

39. Ginn JC, Brener I, Peters DW, Wendt JR, Stevens JO, Hines PF, et al. Realizing optical magnetism from dielectric metamaterials. Phys Rev Lett. (2012) 108(9): 097402. doi:10.1103/PhysRevLett.108.097402

40. Gorkunov MV, Rogov OY, Kondratov AV, Artemov VV, Gainutdinov RV, Ezhov AA. Chiral visible light metasurface patterned in monocrystalline silicon by focused ion beam. Sci Rep. (2018) 8(1):11623. doi:10.1038/ s41598-018-29977-4

41. Grimberg R. Electromagnetic metamaterials. Mater Sci Eng, B. (2013) 178(19): 1285-95. doi:10.1016/j.mseb.2013.03.022

42. Gu J, Singh R, Liu X, Zhang X, Ma Y, Zhang S, et al. Active control of electromagnetically induced transparency analogue in terahertz metamaterials. Nat Commun. (2012) 3:1151. doi:10.1038/ncomms2153

43. Guo X, Pu M, Guo Y, Ma X, Li X, Luo X. Flexible and tunable dielectric color meta-hologram. Plasmonics. (2019) 15(1):217-23. doi:10.1007/s11468-01901025-5

44. Guo Y, Yan L, Pan W, Shao L. Scattering engineering in continuously shaped metasurface: an approach for electromagnetic illusion. Sci Rep. (2016) 6:30154. doi:10.1038/srep30154

45. Gutruf P, Zou C, Withayachumnankul W, Bhaskaran M, Sriram S, Fumeaux C. Mechanically tunable dielectric resonator metasurfaces at visible frequencies. ACS Nano. (2016) 10(1):133-41. doi:10.1021/acsnano.5b05954

46. High AA, Devlin RC, Dibos A, Polking M, Wild DS, Perczel J, et al. Visiblefrequency hyperbolic metasurface. Nature. (2015) 522(7555):192-6. doi:10. 1038/nature14477

47. Hirai Y, Tanaka Y. Application of nano-imprint lithography. J Photopolym Sci Technol. (2002) 15(3):475-80. doi:10.2494/photopolymer.15.475

48. Hou B, Xu G, Wong H, Wen W. Tuning of photonic bandgaps by a fieldinduced structural change of fractal metamaterials. Opt Express. (2005) 13(23): 9149-54. doi:10.1364/opex.13.009149

49. Hsiao H-H, Chu CH, Tsai DP. Fundamentals and applications of metasurfaces. Small Methods. (2017) 1(4). doi:10.1002/smtd.201600064

50. Hsu L, Ndao A, Kante B. Broadband and linear polarization metasurface carpet cloak in the visible. Opt Lett. (2019) 44(12):2978-81. doi:10.1364/OL.44 002978

51. Hu J, Lang T, Hong Z, Shen C, Shi G. Comparison of electromagnetically induced transparency performance in metallic and all-dielectric metamaterials. J Lightwave Technol. (2018) 36(11):2083-93. doi:10.1109/jlt. 2018.2804336

52. Hu J, Lang T, Shen C, Shao L. Combined Mie resonance metasurface for wideband terahertz absorber. Appl Sci. (2018) 8(9):1679. doi:10.3390/ app8091679

53. Hu J, Lang T, Shi GH. Simultaneous measurement of refractive index and temperature based on all-dielectric metasurface. Opt Express. (2017) 25(13): 15241-51. doi:10.1364/OE.25.015241 
54. Hu J, Lang T, Xu W, Liu J, Hong Z. Experimental demonstration of electromagnetically induced transparency in a conductively coupled flexible metamaterial with cheap aluminum foil. Nanoscale Res Lett. (2019) 14(1):359. doi:10.1186/s11671-019-3180-y

55. Hu J, Li L, Lin H, Zhang P, Zhou W, Ma Z. Flexible integrated photonics: where materials, mechanics and optics meet [Invited]. Opt Mater Express. (2013) 3(9):1313. doi:10.1364/ome.3.001313

56. Hu X, Xu G, Wen L, Wang H, Zhao Y, Zhang Y, et al. Metamaterial absorber integrated microfluidic terahertz sensors. Laser Photon Rev. (2016) 10(6): 962-9. doi:10.1002/lpor.201600064

57. Huang L, Chen X, Muhlenbernd H, Li G, Bai B, Tan Q, et al. Dispersionless phase discontinuities for controlling light propagation. Nano Lett. (2012) 12(11):5750-5. doi:10.1021/nl303031j

58. Huang L, Chen X, Mühlenbernd H, Zhang H, Chen S, Bai B, et al. Threedimensional optical holography using a plasmonic metasurface. Nat Commun. (2013) 4(1):2808. doi:10.1038/ncomms3808

59. Huang ST, Hsu SF, Tang KY, Yen TJ, Yao DJ. Application of a terahertz system combined with an X-shaped metamaterial microfluidic cartridge. Micromachines. (2020) 11(1):74. doi:10.3390/mi11010074

60. Huang YW, Chen WT, Tsai WY, Wu PC, Wang CM, Sun G, et al. Aluminum plasmonic multicolor meta-hologram. Nano Lett. (2015) 15(5):3122-7. doi:10. 1021/acs.nanolett.5b00184

61. Islam MS, Sultana J, Biabanifard M, Vafapour Z, Nine MJ, Dinovitser A, et al. Tunable localized surface plasmon graphene metasurface for multiband superabsorption and terahertz sensing. Carbon. (2020) 158:559-67. doi:10. 1016/j.carbon.2019.11.026

62. Jakšić Z, Vasiljević-Radović D, Maksimović M, Sarajlić M, Vujanić A, Djurić Z. Nanofabrication of negative refractive index metasurfaces. Microelectron Eng. (2006) 83(4-9):1786-91. doi:10.1016/j.mee.2006.01.197

63. Jang M, Horie Y, Shibukawa A, Brake J, Liu Y, Kamali SM, et al. Wavefront shaping with disorder-engineered metasurfaces. Nat Photon. (2018) 12:84-90. doi:10.1038/s41566-017-0078-Z

64. Jiang L, Zeng S, Xu Z, Ouyang Q, Zhang DH, Chong PHJ, et al. Multifunctional hyperbolic nanogroove metasurface for submolecular detection. Small. (2017) 13(30):1700600. doi:10.1002/smll.201700600

65. Juhl M, Mueller JPB, Leosson K. Metasurface polarimeter on optical fiber facet by nano-transfer to UV-curable hybrid polymer. IEEE J Sel Top Quant Electron. (2019) 25(3):1-7. doi:10.1109/jstqe.2019.2893757

66. Kamali SM, Arbabi A, Arbabi E, Horie Y, Faraon A. Decoupling optical function and geometrical form using conformal flexible dielectric metasurfaces. Nat Commun. (2016) 7:11618. doi:10.1038/ncomms11618

67. Kamali SM, Arbabi E, Arbabi A, Faraon A. A review of dielectric optical metasurfaces for wavefront control. Nanophotonics. (2018) 7(6):1041-68. doi:10.1515/nanoph-2017-0129

68. Kamali SM, Arbabi E, Arbabi A, Horie Y, Faraon A. Highly tunable elastic dielectric metasurface lenses. Laser Photon Rev. (2016) 10(6):1002-8. doi:10. 1002/lpor.201600144

69. Kang L, Jenkins RP, Werner DH. Recent progress in active optical metasurfaces. Adv Opt Mater. (2019) 7(14):1801813. doi:10.1002/adom.201801813

70. Kasirga TS, Ertas YN, Bayindir M. Microfluidics for reconfigurable electromagnetic metamaterials. Appl Phys Lett. (2009) 95(21):214102. doi:10.1063/1.3268448

71. Khorasaninejad M, Aieta F, Kanhaiya P, Kats MA, Genevet P, Rousso D, et al. Achromatic metasurface lens at telecommunication wavelengths. Nano Lett. (2015) 15(8):5358-62. doi:10.1021/acs.nanolett.5b01727

72. Khorasaninejad M, Chen WT, Devlin RC, Oh J, Zhu AY, Capasso F. Metalenses at visible wavelengths: diffraction-limited focusing and subwavelength resolution imaging. Science. (2016a) 352(6290):1190-4. doi:10.1126/science.aaf6644

73. Khorasaninejad M, Zhu AY, Roques-Carmes C, Chen WT, Oh J, Mishra I, et al. Polarization-insensitive metalenses at visible wavelengths. Nano Lett. (2016b) 16(11):7229-34. doi:10.1021/acs.nanolett.6b03626

74. Kildishev AV, Boltasseva A, Shalaev VM. Planar photonics with metasurfaces. Science. (2013) 339(6125):1232009. doi:10.1126/science. 1232009

75. Kim HK, Lee D, Lim S. A fluidically tunable metasurface absorber for flexible large-scale wireless ethanol sensor applications. Sensors. (2016) 16(8):1246. doi:10.3390/s16081246
76. Kim Y, Yeom B, Arteaga O, Jo Yoo S, Lee SG, Kim JG, et al. Reconfigurable chiroptical nanocomposites with chirality transfer from the macro- to the nanoscale. Nat Mater. (2016) 15(4):461-8. doi:10.1038/nmat4525

77. Kim Y, Yuk H, Zhao R, Chester SA, Zhao X. Printing ferromagnetic domains for untethered fast-transforming soft materials. Nature. (2018) 558(7709): 274-9. doi:10.1038/s41586-018-0185-0

78. Koshelev K, Tang Y, Li K, Choi D-Y, Li G, Kivshar Y. Nonlinear metasurfaces governed by bound states in the continuum. ACS Photonics. (2019) 6(7): 1639-44. doi:10.1021/acsphotonics.9b00700

79. Lawrence N, Trevino J, Dal Negro L. Aperiodic arrays of active nanopillars for radiation engineering. J Appl Phys. (2012) 111(11):113101. doi:10.1063/1. 4723564

80. Lee J, Tymchenko M, Argyropoulos C, Chen PY, Lu F, Demmerle F, et al. Giant nonlinear response from plasmonic metasurfaces coupled to intersubband transitions. Nature. (2014) 511(7507):65-9. doi:10.1038/nature13455

81. Leonhardt U. Optical conformal mapping. Science. (2006) 312(5781):1777-80. doi:10.1126/science. 1126493

82. Li J, Yu P, Zhang S, Liu N. Electrically-controlled digital metasurface device for light projection displays. Nat Commun. (2020) 11(1):3574. doi:10.1038/ s41467-020-17390-3

83. Li L, Jun Cui T, Ji W, Liu S, Ding J, Wan X, et al. Electromagnetic reprogrammable coding-metasurface holograms. Nat Commun. (2017) 8(1): 197. doi:10.1038/s41467-017-00164-9

84. Li L, Lin H, Qiao S, Huang YZ, Li JY, Michon J, et al. Monolithically integrated stretchable photonics. Light Sci Appl. (2018a) 7:17138. doi:10.1038/lsa.2017. 138

85. Li L, Lin H, Qiao S, Zou Y, Danto S, Richardson K, et al. Integrated flexible chalcogenide glass photonic devices. Nat Photon. (2014) 8(8):643-9. doi:10. 1038/nphoton.2014.138

86. Li L, Liu Z, Ren X, Wang S, Su VC, Chen MK, et al. Metalens-array-based highdimensional and multiphoton quantum source. Science. (2020) 368(6498): 1487-90. doi:10.1126/science.aba9779

87. Li L, Ruan H, Liu C, Li Y, Shuang Y, Alu A, et al. Machine-learning reprogrammable metasurface imager. Nat Commun. (2019) 10(1):1082. doi:10.1038/s41467-019-09103-2

88. Li P, Dolado I, Alfaro-Mozaz FJ, Casanova F, Hueso LE, Liu S, et al. Infrared hyperbolic metasurface based on nanostructured van der Waals materials. Science. (2018) 359(6378):892-6. doi:10.1126/science.aaq1704

89. Li S, Zhou C, Ban G, Wang H, Lu H, Wang Y. Active all-dielectric bifocal metalens assisted by germanium antimony telluride. J Phys Appl Phys. (2019) 52(9):9. doi:10.1088/1361-6463/aaf7f3

90. Li X, Chen L, Li Y, Zhang X, Pu M, Zhao Z, et al. Multicolor 3D metaholography by broadband plasmonic modulation. Sci Adv. (2016) 2(11): e1601102. doi:10.1126/sciadv. 1601102

91. Li X, Ren H, Chen X, Liu J, Li Q, Li C, et al. Athermally photoreduced graphene oxides for three-dimensional holographic images. Nat Commun. (2015) 6: 6984. doi:10.1038/ncomms7984

92. Li X, Xiao S, Cai B, He Q, Cui TJ, Zhou L. Flat metasurfaces to focus electromagnetic waves in reflection geometry. Opt Lett. (2012) 37(23): 4940-2. doi:10.1364/OL.37.004940

93. Lin D, Fan P, Hasman E, Brongersma ML. Dielectric gradient metasurface optical elements. Science. (2014) 345(6194):298-302. doi:10.1126/science. 1253213

94. Lin RJ, Su VC, Wang S, Chen MK, Chung TL, Chen YH, et al. Achromatic metalens array for full-colour light-field imaging. Nat Nanotechnol. (2019) 14(3):227-31. doi:10.1038/s41565-018-0347-0

95. Ling K, Kim HK, Yoo M, Lim S. Frequency-switchable metamaterial absorber injecting eutectic gallium-indium (EGaIn) liquid metal alloy. Sensors. (2015) 15(11):28154-65. doi:10.3390/s151128154

96. Liu L, Zhang X, Kenney M, Su X, Xu N, Ouyang C, et al. Broadband metasurfaces with simultaneous control of phase and amplitude. Adv Mater. (2014) 26(29):5031-6. doi:10.1002/adma.201401484

97. Liu W, Shen Y, Xiao G, She X, Wang J, Jin C. Mechanically tunable sub-10 nm metal gap by stretching PDMS substrate. Nanotechnology. (2017) 28(7): 075301. doi:10.1088/1361-6528/aa5366

98. Liu X, Fan K, Shadrivov IV, Padilla WJ. Experimental realization of a terahertz all-dielectric metasurface absorber. Opt Express. (2017) 25(1):191-201. doi:10. 1364/OE.25.000191 
99. Liu X, Huang Z, Zhu C, Wang L, Zang J. Out-of-Plane designed soft metasurface for tunable surface plasmon polariton. Nano Lett. (2018) 18(2):1435-41. doi:10.1021/acs.nanolett.7b05190

100. Liu Y, Song J, Zhao W, Ren X, Cheng Q, Luo X, et al. Dynamic thermal camouflage via a liquid-crystal-based radiative metasurface. Nanophotonics. (2020) 9(4):855-63. doi:10.1515/nanoph-2019-0485

101. Luo J, Zeng B, Wang C, Gao P, Liu K, Pu M, et al. Fabrication of anisotropically arrayed nano-slots metasurfaces using reflective plasmonic lithography. Nanoscale. (2015) 7(44):18805-12. doi:10.1039/ c5nr05153c

102. Luo W, Sun S, Xu H-X, He Q, Zhou L. Transmissive ultrathin pancharatnamberry metasurfaces with nearly 100\% efficiency. Phys Rev Appl. (2017) 7(4): 044033. doi:10.1103/PhysRevApplied.7.044033

103. Ma Q, Bai GD, Jing HB, Yang C, Li L, Cui TJ. Smart metasurface with selfadaptively reprogrammable functions. Light Sci Appl. (2019) 8:98. doi:10. 1038/s41377-019-0205-3

104. Maci S, Minatti G, Casaletti M, Bosiljevac M. Metasurfing: addressing waves on impenetrable metasurfaces. IEEE Antenn Wireless Propag Lett. (2011) 10: 1499-502. doi:10.1109/lawp.2012.2183631

105. Makarov SV, Milichko V, Ushakova EV, Omelyanovich M, Cerdan Pasaran A, Haroldson R, et al. Multifold emission enhancement in nanoimprinted hybrid perovskite metasurfaces. ACS Photonics. (2017) 4(4):728-35. doi:10. 1021/acsphotonics.6b00940

106. Mark D, Haeberle S, Roth G, von Stetten F, Zengerle R. Microfluidic lab-on-achip platforms: requirements, characteristics and applications. Chem Soc Rev. (2010) 39(3):1153-82. doi:10.1039/b820557b

107. Meinzer N, Barnes WL, Hooper IR. Plasmonic meta-atoms and metasurfaces. Nat Photon. (2014) 8(12):889-98. doi:10.1038/nphoton.2014.247

108. Mohammadi Estakhri N, Alù A. Manipulating optical reflections using engineered nanoscale metasurfaces. Phys Rev B. (2014) 89(23):235419. doi:10.1103/PhysRevB.89.235419

109. Monticone F, Estakhri NM, Alu A. Full control of nanoscale optical transmission with a composite metascreen. Phys Rev Lett. (2013) 110(20): 203903. doi:10.1103/PhysRevLett.110.203903

110. Nemiroski A, Gonidec M, Fox JM, Jean-Remy P, Turnage E, Whitesides GM. Engineering shadows to fabricate optical metasurfaces. ACS Nano. (2014) 8(11):11061-70. doi:10.1021/nn504214b

111. Ni X, Emani NK, Kildishev AV, Boltasseva A, Shalaev VM. Broadband light bending with plasmonic nanoantennas. Science. (2012) 335(6067):427. doi:10.1126/science. 1214686

112. Ni X, Ishii S, Kildishev AV, Shalaev VM. Ultra-thin, planar, Babinet-inverted plasmonic metalenses. Light Sci Appl. (2013) 2(4):e72. doi:10.1038/lsa.2013.28

113. Ni X, Kildishev AV, Shalaev VM. Metasurface holograms for visible light. Nat Commun. (2013) 4(1):2807. doi:10.1038/ncomms3807

114. Peng R, Liu J, Xiao D, Fang X, Yin S, Yin Z, et al. Microfluid-enabled fine tuning of circular dichroism from chiral metasurfaces. J Phys Appl Phys. (2019) 52(41):415102. doi:10.1088/1361-6463/ab3129

115. Person S, Jain M, Lapin Z, Saenz JJ, Wicks G, Novotny L. Demonstration of zero optical backscattering from single nanoparticles. Nano Lett. (2013) 13(4):1806-9. doi:10.1021/nl4005018

116. Pfeiffer C, Grbic A. Metamaterial Huygens' surfaces: tailoring wave fronts with reflectionless sheets. Phys Rev Lett. (2013) 110(19):197401. doi:10.1103/ PhysRevLett.110.197401

117. Pimenov A, Loidl A. Experimental demonstration of artificial dielectrics with a high index of refraction. Phys Rev B. (2006) 74(19):193108. doi:10.1103/ PhysRevB.74.193102

118. Pisco M, Galeotti F, Quero G, Grisci G, Micco A, Mercaldo LV, et al. Nanosphere lithography for optical fiber tip nanoprobes. Light Sci Appl. (2017) 6(5):e16229. doi:10.1038/lsa.2016.229

119. Pisco M, Galeotti F, Quero G, Iadicicco A, Giordano M, Cusano A. Miniaturized sensing probes based on metallic dielectric crystals selfassembled on optical fiber tips. ACS Photonics. (2014) 1(10):917-27. doi:10.1021/ph500126v

120. Pitchappa P, Manjappa M, Ho CP, Singh R, Singh N, Lee C. Active control of electromagnetically induced transparency analog in terahertz MEMS metamaterial. Adv Opt Mater. (2016) 4(4):541-7. doi:10.1002/adom. 201500676
121. Pors A, Albrektsen O, Radko IP, Bozhevolnyi SI. Gap plasmon-based metasurfaces for total control of reflected light. Sci Rep. (2013) 3:2155. doi:10.1038/srep02155

122. Pors A, Bozhevolnyi SI. Plasmonic metasurfaces for efficient phase control in reflection. Opt Express. (2013) 21(22):27438-51. doi:10.1364/oe.21.027438

123. Pors A, Nielsen MG, Eriksen RL, Bozhevolnyi SI. Broadband focusing flat mirrors based on plasmonic gradient metasurfaces. Nano Lett. (2013) 13(2): 829-34. doi:10.1021/nl304761m

124. Principe M, Consales M, Castaldi G, Galdi V, Cusano A. Evaluation of fiberoptic phase-gradient meta-tips for sensing applications. Nanomater Nanotechnol. (2019) 9. doi:10.1177/1847980419832724

125. Principe M, Consales M, Micco A, Crescitelli A, Castaldi G, Esposito E, et al. Optical fiber meta-tips. Light Sci Appl. (2017) 6(3):e16226. doi:10.1038/lsa. 2016.226

126. Qian C, Zheng B, Shen Y, Jing L, Li E, Shen L, et al. Deep-learning-enabled self-adaptive microwave cloak without human intervention. Nat Photon. (2020) 14(6):383-90. doi:10.1038/s41566-020-0604-2

127. Rocco D, Carletti L, Caputo R, Finazzi M, Celebrano M, De Angelis C. Switching the second harmonic generation by a dielectric metasurface via tunable liquid crystal. Opt Express. (2020) 28(8):12037-46. doi:10.1364/OE. 386776

128. Roy T, Zhang S, Jung IW, Troccoli M, Capasso F, Lopez D. Dynamic metasurface lens based on MEMS technology. APL Photonics. (2018) 3(2): 021302. doi:10.1063/1.5018865

129. Ruiz de Galarreta C, Sinev I, Alexeev AM, Trofimov P, Ladutenko K, GarciaCuevas Carrillo S, et al. Reconfigurable multilevel control of hybrid alldielectric phase-change metasurfaces. Optica. (2020) 7(5):476. doi:10.1364/ optica. 384138

130. Sain B, Meier C, Zentgraf T. Nonlinear optics in all-dielectric nanoantennas and metasurfaces: a review. Adv Photonics. (2019) 1(02):024002. doi:10.1117/ 1.Ap.1.2.024002

131. Scheerlinck S, Dubruel P, Bienstman P, Schacht E, Van Thourhout D, Baets R. Metal grating patterning on fiber facets by UV-based nano imprint and transfer lithography using optical alignment. J Lightwave Technol. (2009) 27(10):1415-20. doi:10.1109/jlt.2008.2004955

132. Schift H, Park S, Gobrecht J. Nano-imprint-molding resists for lithography. J Photopolym Sci Technol. (2003) 16(3):435-8. doi:10.2494/photopolymer.16. 435

133. Selvanayagam M, Eleftheriades GV. Discontinuous electromagnetic fields using orthogonal electric and magnetic currents for wavefront manipulation. Opt Express. (2013) 21(12):14409-29. doi:10.1364/OE.21.014409

134. Shalaev MI, Sun J, Tsukernik A, Pandey A, Nikolskiy K, Litchinitser NM. High-efficiency all-dielectric metasurfaces for ultracompact beam manipulation in transmission mode. Nano Lett. (2015) 15(9):6261-6. doi:10.1021/acs.nanolett.5b02926

135. Sharma M, Hendler N, Ellenbogen T. Electrically switchable color tags based on active liquid-crystal plasmonic metasurface platform. Adv Opt Mater. (2020) 8(7). doi:10.1002/adom.201901182

136. Shen JT, Catrysse PB, Fan S. Mechanism for designing metallic metamaterials with a high index of refraction. Phys Rev Lett. (2005) 94(19):197401. doi:10. 1103/PhysRevLett.94.197401

137. Shrestha S, Overvig AC, Lu M, Stein A, Yu N. Broadband achromatic dielectric metalenses. Light Sci Appl. (2018) 7:85. doi:10.1038/s41377-0180078-x

138. Sirmaci YD, Tang Z, Fasold S, Neumann C, Pertsch T, Turchanin A, et al. Plasmonic metasurfaces situated on ultrathin carbon nanomembranes. ACS Photonics. (2020) 7(4):1060-6. doi:10.1021/acsphotonics.0c00073

139. Smith DR, Pendry JB, Wiltshire MC Metamaterials and negative refractive index. Science. (2004) 305(5685):788-92. doi:10.1126/science.1096796

140. Su VC, Chu $\mathrm{CH}$, Sun G, Tsai DP Advances in optical metasurfaces: fabrication and applications [Invited]. Opt Express. (2018) 26(10): 13148-82. doi:10.1364/OE.26.013148

141. Sun S, Yang KY, Wang CM, Juan TK, Chen WT, Liao CY, et al. Highefficiency broadband anomalous reflection by gradient meta-surfaces. Nano Lett. (2012) 12(12):6223-9. doi:10.1021/nl3032668

142. Valentine J, Li J, Zentgraf T, Bartal G, Zhang X An optical cloak made of dielectrics. Nat Mater. (2009) 8(7):568-71. doi:10.1038/nmat2461 
143. Valentine J, Zhang S, Zentgraf T, Ulin-Avila E, Genov DA, Bartal G, et al. Three-dimensional optical metamaterial with a negative refractive index. Nature. (2008) 455(7211):376-9. doi:10.1038/nature07247

144. Walia S, Shah CM, Gutruf P, Nili H, Chowdhury DR, Withayachumnankul $\mathrm{W}$, et al. Flexible metasurfaces and metamaterials: a review of materials and fabrication processes at micro- and nano-scales. Appl Phys Rev. (2015) 2(1): 011303. doi:10.1063/1.4913751

145. Wan W, Gao J, Yang X Full-color plasmonic metasurface holograms. ACS Nano. (2016) 10(12):10671-80. doi:10.1021/acsnano.6b05453

146. Wan W, Gao J, Yang X Metasurface holograms for holographic imaging. Adv Opt Mater. (2017) 5(21):011303. doi:10.1002/adom.201700541

147. Wang B, Dong F, Li QT, Yang D, Sun C, Chen J, et al. Visible-frequency dielectric metasurfaces for multiwavelength achromatic and highly dispersive holograms. Nano Lett. (2016) 16(8):5235-40. doi:10.1021/acs.nanolett.6b02326

148. Wang C, Yang Y, Liu Q, Liang D, Zheng B, Chen H, et al. Multi-frequency metasurface carpet cloaks. Opt Express. (2018) 26(11):14123-31. doi:10.1364/ OE.26.014123

149. Wang H, Liu SC, Balachandran B, Moon J, Haroldson R, Li Z, et al. Nanoimprinted perovskite metasurface for enhanced photoluminescence. Opt Express. (2017) 25(24):A1162-A1171. doi:10.1364/OE.25.0A1162

150. Wang J, Liu S, Guruswamy S, Nahata A Reconfigurable terahertz metamaterial device with pressure memory. Opt Express. (2014) 22(4): 4065-74. doi:10.1364/OE.22.004065

151. Wang J, Liu S, Vardeny ZV, Nahata A Liquid metal-based plasmonics. Opt Express. (2012) 20(3):2346-53. doi:10.1364/OE.20.002346

152. Wang L, Kruk S, Tang H, Li T, Kravchenko I, Neshev DN, et al. Grayscale transparent metasurface holograms. Optica. (2016) 3(12):1504. doi:10.1364/ optica.3.001504

153. Wang L, Liu X, Zang J Mechanically tunable terahertz graphene plasmonics using soft metasurface. 2D Mater. (2016) 3(4):041007. doi:10.1088/2053$1583 / 3 / 4 / 041007$

154. Wang Q, Rogers ETF, Gholipour B, Wang C-M, Yuan G, Teng J, et al. Optically reconfigurable metasurfaces and photonic devices based on phase change materials. Nat Photon. (2015) 10(1):60-5. doi:10.1038/nphoton.2015.247

155. Wang S, Wu PC, Su VC, Lai YC, Chen MK, Kuo HY, et al. A broadband achromatic metalens in the visible. Nat Nanotechnol. (2018) 13(3):227-32. doi:10.1038/s41565-017-0052-4

156. Wang S, Wu PC, Su VC, Lai YC, Hung Chu C, Chen JW, et al. Broadband achromatic optical metasurface devices. Nat Commun. (2017) 8(1):187. doi:10.1038/s41467-017-00166-7

157. Wang Y, Liu F, Zhang X Flexible transfer of plasmonic photonic structures onto fiber tips for sensor applications in liquids. Nanoscale. (2018) 10(34): 16193-200. doi:10.1039/c8nr05871g

158. Wei M, Yang Q, Zhang X, Li Y, Gu J, Han J, et al. Ultrathin metasurface-based carpet cloak for terahertz wave. Opt Express. (2017) 25(14):15635-42. doi:10. 1364/OE.25.015635

159. Wu J, Shen Z, Ge S, Chen B, Shen Z, Wang T, et al. Liquid crystal programmable metasurface for terahertz beam steering. Appl Phys Lett. (2020) 116(13):131104. doi:10.1063/1.5144858

160. Xiao D, Liu YJ, Yin S, Liu J, Ji W, Wang B, et al. Liquid-crystal-loaded chiral metasurfaces for reconfigurable multiband spin-selective light absorption. Opt Express. (2018) 26(19):25305-14. doi:10.1364/OE.26.025305

161. Xiaofang Su XS, Li G, Yang H, Zhao Z, Yu F, Chen X, et al. A visible high efficiency and polarization-insensitive 34-level dielectric metasurface hologram. RSC Adv. (2017) 7(42):26371-6. doi:10. 1039/c7ra03672h

162. Xie Y, Wang W, Chen H, Konneker A, Popa BI, Cummer SA Wavefront modulation and subwavelength diffractive acoustics with an acoustic metasurface. Nat Commun. (2014) 5:5553. doi:10.1038/ncomms6553

163. Xomalis A, Demirtzioglou I, Plum E, Jung Y, Nalla V, Lacava C, et al. Fibre-optic metadevice for all-optical signal modulation based on coherent absorption. Nat Commun. (2018) 9(1):182. doi:10.1038/ s41467-017-02434-y

164. Xu J, Fan Y, Yang R, Fu Q, Zhang F Realization of switchable EIT metamaterial by exploiting fluidity of liquid metal. Opt Express. (2019) 27(3):2837-43. doi:10.1364/OE.27.002837

165. Xu Y, Fu Y, Chen H Planar gradient metamaterials. Nat Rev Mater. (2016) 1(12):16067. doi:10.1038/natrevmats.2016.67
166. Yan LB, Zhu WM, Wu PC, Cai H, Gu YD, Chin LK, et al. Adaptable metasurface for dynamic anomalous reflection. Appl Phys Lett. (2017) 110(20):201904. doi:10.1063/1.4983782

167. Yang Y, KravchenkoII, Briggs DP, Valentine J All-dielectric metasurface analogue of electromagnetically induced transparency. Nat Commun. (2014) 5:5753. doi:10.1038/ncomms6753

168. Yang Y, Wang W, Moitra P, Kravchenko II, Briggs DP, Valentine J Dielectric meta-reflectarray for broadband linear polarization conversion and optical vortex generation. Nano Lett. (2014) 14(3):1394-9. doi:10.1021/nl4044482

169. Yao Y, Shankar R, Kats MA, Song Y, Kong J, Loncar M, et al. Electrically tunable metasurface perfect absorbers for ultrathin mid-infrared optical modulators. Nano Lett. (2014) 14(11):6526-32. doi:10.1021/nl503104n

170. Ye W, Zeuner F, Li X, Reineke B, He S, Qiu CW, et al. Spin and wavelength multiplexed nonlinear metasurface holography. Nat Commun. (2016) 7: 11930. doi:10.1038/ncomms 11930

171. Yin S, Ji W, Xiao D, Li Y, Li K, Yin Z, et al. Intrinsically or extrinsically reconfigurable chirality in plasmonic chiral metasurfaces. Optic Commun. (2019) 448:10-4. doi:10.1016/j.optcom.2019.05.006

172. Yin S, Xiao D, Liu J, Li K, He H, Jiang S, et al. Reconfigurable chiral metasurface absorbers based on liquid crystals. IEEE Photonics J. (2018) 10(6):1-9. doi:10.1109/jphot.2018.2878775

173. Yoon G, Kim K, Huh D, Lee H, Rho J Single-step manufacturing of hierarchical dielectric metalens in the visible. Nat Commun. (2020) 11(1): 2268. doi:10.1038/s41467-020-16136-5

174. Yu N, Capasso F Flat optics with designer metasurfaces. Nat Mater. (2014) 13(2):139-50. doi:10.1038/nmat3839

175. Yu N, Genevet P, Aieta F, Kats MA, Blanchard R, Aoust G, et al. Flat optics: controlling wavefronts with optical antenna metasurfaces. IEEE J Sel Top Quant Electron. (2013) 19(3):4700423. doi:10.1109/jstqe.2013.2241399

176. Yu N, Genevet P, Kats MA, Aieta F, Tetienne JP, Capasso F, et al. Light propagation with phase discontinuities: generalized laws of reflection and refraction. Science. (2011) 334(6054):333-7. doi:10.1126/science. 1210713

177. Yu YF, Zhu AY, Paniagua-Domínguez R, Fu YH, Luk'yanchuk B, Kuznetsov AI High-transmission dielectric metasurface with $2 \pi$ phase control at visible wavelengths. Laser Photon Rev. (2015) 9(4):412-8. doi:10.1002/lpor.201500041

178. Yue F, Wen D, Xin J, Gerardot BD, Li J, Chen X Vector vortex beam generation with a single plasmonic metasurface. ACS Photonics. (2016) 3(9): 1558-63. doi:10.1021/acsphotonics.6b00392

179. Zeng J, Li L, Yang X, Gao J Generating and separating twisted light by gradient-rotation split-ring antenna metasurfaces. Nano Lett. (2016) 16(5): 3101-8. doi:10.1021/acs.nanolett.6b00360

180. Zhang H-F, Wang Z-L, Hu C-X, Liu H-B A tailored broadband terahertz metamaterial absorber based on the thermal expansion feature of liquid metal. Results Phys. (2020) 16:102936. doi:10.1016/j.rinp.2020.102937

181. Zhang H, Feng L, Liang Y, Xu T An ultra-flexible plasmonic metamaterial film for efficient omnidirectional and broadband optical absorption. Nanoscale. (2019) 11(2):437-43. doi:10.1039/c8nr05276j

182. Zhang M, Zhang W, Liu AQ, Li FC, Lan CF Tunable polarization conversion and rotation based on a reconfigurable metasurface. Sci Rep. (2017) 7(1): 12068. doi:10.1038/s41598-017-11953-z

183. Zhang S, Kim MH, Aieta F, She A, Mansuripur T, Gabay I, et al. High efficiency near diffraction-limited mid-infrared flat lenses based on metasurface reflectarrays. Opt Express. (2016) 24(16):18024-34. doi:10. 1364/OE.24.018024

184. Zhang W, Song Q, Zhu W, Shen Z, Chong P, Tsai DP, et al. Metafluidic metamaterial: a review. Adv Phys X. (2018) 3(1):1417055. doi:10.1080/ 23746149.2017.1417055

185. Zhang Y, Liang L, Yang J, Feng Y, Zhu B, Zhao J, et al. Broadband diffuse terahertz wave scattering by flexible metasurface with randomized phase distribution. Sci Rep. (2016) 6:26875. doi:10.1038/srep26875

186. Zhang Z, Luo J, Song M, Yu H Large-area, broadband and high-efficiency near-infrared linear polarization manipulating metasurface fabricated by orthogonal interference lithography. Appl Phys Lett. (2015) 107(24): 241904. doi:10.1063/1.4937006

187. Zhao J, Sima B, Jia N, Wang C, Zhu B, Jiang T, et al. Achieving flexible lowscattering metasurface based on randomly distribution of meta-elements. Opt Express. (2016) 24(24):27849-57. doi:10.1364/OE.24.027849 
188. Zhao W, Jiang H, Liu B, Song J, Jiang Y, Tang C, et al. Dielectric Huygens' metasurface for high-efficiency hologram operating in transmission mode. Sci Rep. (2016) 6:30613. doi:10.1038/srep30613

189. Zhao W, Li X, Li S, Ge Z, Liu M, Li Z, et al. Sub-wavelength focusing based on all-dielectric polarization-independent metalens. Int J Mod Phys B. (2018) 32(29):18321. doi:10.1142/s0217979218503216

190. Zhao X, Schalch J, Zhang J, Seren HR, Duan G, Averitt RD, et al. Electromechanically tunable metasurface transmission waveplate at terahertz frequencies. Optica. (2018) 5(3):303. doi:10.1364/optica.5. 000303

191. Zhao Y, Zhang J, Du J, Wang J Meta-facet fiber for twisting ultra-broadband light with high phase purity. Appl Phys Lett. (2018) 113(6):061103. doi:10. $1063 / 1.5043268$

192. Zheludev NI, Kivshar YS From metamaterials to metadevices. Nat Mater. (2012) 11(11):917-24. doi:10.1038/nmat3431

193. Zheng G, Muhlenbernd H, Kenney M, Li G, Zentgraf T, Zhang S Metasurface holograms reaching 80\% efficiency. Nat Nanotechnol. (2015) 10(4):308-12. doi:10.1038/nnano.2015.2

194. Zhu HL, Cheung SW, Chung KL, Yuk TI Linear-to-circular polarization conversion using metasurface. IEEE Trans Antenn Propag. (2013) 61(9): 4615-23. doi:10.1109/tap.2013.2267712
195. Zhu Y, Li Z, Hao Z, DiMarco C, Maturavongsadit P, Hao Y, et al. Optical conductivity-based ultrasensitive mid-infrared biosensing on a hybrid metasurface. Light Sci Appl. (2018) 7:67. doi:10.1038/s41377-018-0066-1

196. Ziolkowski RW Propagation in and scattering from a matched metamaterial having a zero index of refraction. Phys Rev E-Stat Nonlin Soft Matter Phys. (2004) 70(4):046608. doi:10.1103/PhysRevE.70.046608

197. Zuo R, Liu W, Cheng H, Chen S, Tian J Breaking the diffraction limit with radially polarized light based on dielectric metalenses. Adv Opt Mater. (2018) 6(21):1800795. doi:10.1002/adom.201800795

Conflict of Interest: The authors declare that the research was conducted in the absence of any commercial or financial relationships that could be construed as a potential conflict of interest.

Copyright (C) $2021 \mathrm{Hu}$, Bandyopadhyay, Liu and Shao. This is an open-access article distributed under the terms of the Creative Commons Attribution License (CC BY). The use, distribution or reproduction in other forums is permitted, provided the original author(s) and the copyright owner(s) are credited and that the original publication in this journal is cited, in accordance with accepted academic practice. No use, distribution or reproduction is permitted which does not comply with these terms. 